Florida International University

FIU Digital Commons

FIU Electronic Theses and Dissertations

University Graduate School

6-27-2019

\title{
Unmanned Aerial Vehicles (UAVs) for Integrated Access and Backhaul (IAB) Communications in Wireless Cellular Networks
}

Abdurrahman Fouda

afoud004@fiu.edu

Follow this and additional works at: https://digitalcommons.fiu.edu/etd

Part of the Systems and Communications Commons

\section{Recommended Citation}

Fouda, Abdurrahman, "Unmanned Aerial Vehicles (UAVs) for Integrated Access and Backhaul (IAB) Communications in Wireless Cellular Networks" (2019). FIU Electronic Theses and Dissertations. 4212. https://digitalcommons.fiu.edu/etd/4212

This work is brought to you for free and open access by the University Graduate School at FIU Digital Commons. It has been accepted for inclusion in FIU Electronic Theses and Dissertations by an authorized administrator of FIU Digital Commons. For more information, please contact dcc@fiu.edu. 


\title{
FLORIDA INTERNATIONAL UNIVERSITY \\ Miami, Florida
}

\section{UNMANNED AERIAL VEHICLES (UAVS) FOR INTEGRATED ACCESS AND BACKHAUL (IAB) COMMUNICATIONS IN WIRELESS CELLULAR NETWORKS}

\author{
A thesis submitted in partial fulfillment of the \\ requirements for the degree of \\ MASTER OF SCIENCE \\ in \\ ELECTRICAL ENGINEERING \\ by
}

Abdurrahman Fouda 
To: Dean John L. Volakis

College of Engineering and Computing

This thesis, written by Abdurrahman Fouda, and entitled Unmanned Aerial Vehicles (UAVs) for Integrated Access and Backhaul (IAB) Communications in Wireless Cellular Networks, having been approved in respect to style and intellectual content, is referred to you for judgment.

We have read this thesis and recommend that it be approved.

Armando Barreto

Kemal Akkaya

Elias Alwan

Ahmed S. Ibrahim, Major Professor

Date of Defense: June 27, 2019

The thesis of Abdurrahman Fouda is approved.

Dean John L. Volakis
College of Engineering and Computing

Andrés G. Gil

Vice president for Research and Economic Development and Dean of the University Graduate School

Florida International University, 2019 


\section{ACKNOWLEDGMENTS}

I would like to express my gratitude to my advisor Prof. Ahmed S. Ibrahim for his continuous support and mentorship during my master's thesis. I would like also to thank Prof. İsmail Güvenç and Prof. Monisha Ghosh whose support has helped me to complete this work. Furthermore, I would like to thank the committee members: Prof. Armando Barreto, Prof. Kemal Akkaya and Prof. Elias Alwan for their valuable guidance and feedback that helped me to improve the content of this thesis. 


\title{
ABSTRACT OF THE THESIS \\ UNMANNED AERIAL VEHICLES (UAVS) FOR INTEGRATED ACCESS AND \\ BACKHAUL (IAB) COMMUNICATIONS IN WIRELESS CELLULAR NETWORKS \\ by
}

\author{
Abdurrahman Fouda \\ Florida International University, 2019 \\ Miami, Florida \\ Professor Ahmed S. Ibrahim, Major Professor
}

An integrated access and backhaul (IAB) network architecture can enable flexible and fast deployment of next-generation cellular networks. However, mutual interference between access and backhaul links, small inter-site distance and spatial dynamics of user distribution pose major challenges in the practical deployment of IAB networks. To tackle these problems, we leverage the flying capabilities of unmanned aerial vehicles (UAVs) as hovering IAB-nodes and propose an interference management algorithm to maximize the overall sum rate of the IAB network. In particular, we jointly optimize the user and base station associations, the downlink power allocations for access and backhaul transmissions, and the spatial configurations of UAVs. We consider two spatial configuration modes of UAVs: distributed UAVs and drone antenna array (DAA), and show how they are intertwined with the spatial distribution of ground users. Our numerical results show that the proposed algorithm achieves an average of $2.9 \times$ and $6.7 \times$ gains in the received downlink signal-to-interference-plus-noise ratio (SINR) and overall network sum rate, respectively. Finally, the numerical results reveal that UAVs cannot only be used for coverage improvement but also for capacity boosting in IAB cellular networks. 


\section{TABLE OF CONTENTS}

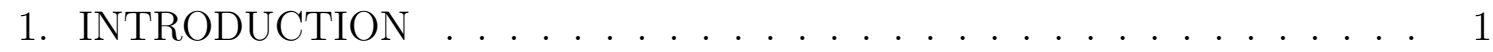

1.1 Motivation . . . . . . . . . . . . . . . . . . . . . . . . . . . 1

1.2 Literature review and related work . . . . . . . . . . . . . . . 3

1.3 Contribution of the Thesis $\ldots \ldots \ldots$. . . . . . . . . . . . . . . . 14

1.4 Organization of the Thesis $\ldots \ldots \ldots \ldots$

1.5 Notation . . . . . . . . . . . . . . . . . . . . . . . . . . . . . 17

2. SYSTEM AND RECEIVED SIGNAL MODEL . . . . . . . . . . . . . . 18

2.1 Generic Channel Model . . . . . . . . . . . . . . . . . . . . . . . . . . . . 19

2.2 Distributed UAVs Spatial Configuration Mode . . . . . . . . . . . . . 20

2.2 .1 Backhaul Downlink Transmissions $\ldots \ldots \ldots$

$2.2 .2 \quad$ Access Downlink Transmissions . . . . . . . . . . . . . . . . . . . 21

2.3 Drone Antenna Array Spatial Configuration Mode. . . . . . . . . . . . . 22

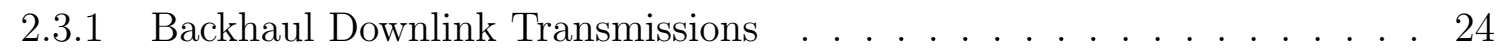

2.3 .2 Access Downlink Transmissions . . . . . . . . . . . . . . . . . 25

3. SUM-RATE MAXIMIZATION PROBLEM IN UAV-ASSISTED IAB CEL-

LULAR NETWORKS . . . . . . . . . . . . . . . . . . 27

3.1 Distributed UAVs Spatial Configuration Mode . . . . . . . . . . . . . . . 27

3.2 Drone Antenna Array Spatial Configuration Mode. . . . . . . . . . . . . . 29

3.3 Hybrid Fixed-Point Iteration and Particle Swarm Approach . . . . . . . 30

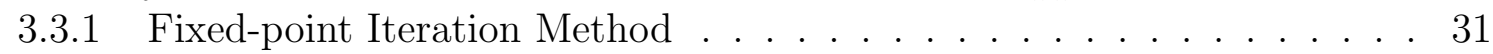

3.3 .2 Particle Swarm Optimization . . . . . . . . . . . . . . . . . . . . . 35

3.3 .3 General Solution . . . . . . . . . . . . . . . . . . . . . . . . . . . . . . 39

4. NUMERICAL RESULTS . . . . . . . . . . . . . . . . . . . . . 41

4.1 Dual Clusters Spatial Distribution of Cellular Users . . . . . . . . . . . . 41

4.2 Multiple Clusters Spatial Distribution of Cellular Users . . . . . . . . . . 45

4.3 Convergence Analysis of the PSO Algorithm . . . . . . . . . . . . . . 48

4.4 Numerical Evaluation of Reversed Algorithm 3. . . . . . . . . . . . . . 50

4.5 Generic Spatial Distribution of Cellular Users . . . . . . . . . . . . . . 51

5. CONCLUDING REMARKS $\ldots \ldots \ldots \ldots \ldots \ldots \ldots$

BIBLIOGRAPHY . . . . . . . . . . . . . . . . . . 57 


\section{LIST OF TABLES}

TABLE

PAGE

3.1 Time complexity of the proposed algorithms . . . . . . . . . . . . . . . 39

$4.1 \quad$ Simulation parameters $\ldots \ldots \ldots \ldots$. . . . . . . . . . . . . . . . . 42 


\section{LIST OF FIGURES}

FIGURE

PAGE

2.1 In-band IAB system architecture for next-generation cellular networks: UAVs can be users themselves or operate as drone IAB-nodes to serve other users. . . . . . . . . . . . . . . . . . . . 19

$2.2 \quad$ Drone antenna array design parameters. . . . . . . . . . . . . . . . . . . 23

$4.1 \quad$ Dual clusters: spatial configurations of DAA. . . . . . . . . . . . . . . . 42

4.2 Dual clusters: received downlink SINR. . . . . . . . . . . . . . . . . . 43

4.3 Dual clusters: received downlink user throughput. . . . . . . . . . . . . 44

4.4 The computational complexity of Algorithm 2 2 with respect to the number of drones per DAA. . . . . . . . . . . . . . . 44

4.5 Multiple clusters: spatial configurations of UAVs. . . . . . . . . . . . . . 46

$4.6 \quad$ Multiple clusters: received downlink SINR. . . . . . . . . . . . . . . . . 46

$4.7 \quad$ Multiple clusters: received downlink user throughput. . . . . . . . . . . 47

$4.8 \quad$ Favorable spatial configurations of UAVs. . . . . . . . . . . . . . . . . . 48

$4.9 \quad$ Dual clusters: PSO convergence. . . . . . . . . . . . . . . . . . . . . . . 49

4.10 Multiple clusters: PSO convergence. . . . . . . . . . . . . . . . . . . . . 50

4.11 Reversed Algorithm 3 : downlink SINR. . . . . . . . . . . . . . . . . . . 51

4.12 Reversed Algorithm|3: downlink throughput. . . . . . . . . . . . . . . . 52

4.13 Generic spatial distribution of cellular users. . . . . . . . . . . . . . . . . 53

4.14 Generic distribution: downlink SINR. . . . . . . . . . . . . . . . . 53

4.15 Coupled distribution: downlink backhaul SINR. . . . . . . . . . . . . . . 54 


\section{LIST OF ABBREVIATIONS}

\begin{tabular}{|c|c|}
\hline 3GPP & 3rd Generation Partnership Project \\
\hline AoD & angle-of-departure \\
\hline ASD & angular spread of departure \\
\hline aUE & aerial user \\
\hline CSI & channel state information \\
\hline DAA & drone antenna array \\
\hline DF & decode-and-forward \\
\hline ETP & equal transmit power \\
\hline HetNets & heterogeneous networks \\
\hline IAB & integrated access and backhaul \\
\hline LOS & line-of-sight \\
\hline LZFBF & linear zero-forcing beamforming \\
\hline MBS & macro base station \\
\hline MISO & multiple-input-single-output \\
\hline NOMA & non-orthogonal multiple access \\
\hline NP-MINLP & NP-hard mixed-integer nonlinear program \\
\hline $\mathrm{PSO}$ & particle swarm optimization \\
\hline QoS & quality of service \\
\hline SBS & small base station \\
\hline SDMA & spatial division multiple access \\
\hline SINR & signal-to-interference-plus-noise ratio \\
\hline tUE & terrestrial user \\
\hline UAV & unmanned aerial vehicle \\
\hline ULA & uniform linear array \\
\hline
\end{tabular}




\section{CHAPTER 1 \\ INTRODUCTION}

\subsection{Motivation}

The exponential increase in capacity and mobile traffic demands has revolutionized the design methodologies of next-generation cellular networks. In recent years, the basic idea of dense deployment of small base stations (SBSs) for capacity boosting and/or coverage enhancement has laid the foundation for the concept of multitier heterogeneous networks (HetNets). Essentially, the dense deployment of SBSs raises the need for easily scalable solutions to meet the requirements of network densification. In typical macro-cell deployments, the high-speed optical fiber can offer reliable communication links and high data rates. Hence, it is considered as an appropriate medium for the backhaul network traffic. On the contrary, wireless backhauling has been emerged as a potential solution for HetNets to reduce the network deployment cost, in which, the macro base station (MBS) provides wireless access and backhauling functionality to cellular users and SBSs respectively [1,2].

In this regard, 3rd Generation Partnership Project (3GPP) has introduced the integrated access and backhaul (IAB) network architecture to allow for flexible deployment of next-generation cellular networks [3,4]. Generally, the IAB architecture implies tight interworking between access and backhaul links, where the IAB-donor (i.e., macro base station (MBS)) uses the same infrastructure and wireless channel resources to provide access and backhauling functionalities for cellular users and IAB-nodes (i.e., small bases stations (SBSs)), respectively [5 7]. Although IAB-based cellular networks are envisioned to meet the increase in user and traffic demands, the mutual interference between access and backhaul links and the limitations of backhaul capacity are among the main challenges to develop reliable 
communication links in IAB networks (see, e.g., [5]). Moreover, the random massive deployment of users and base stations (BSs) poses another challenge in the design of reliable interference management frameworks (user-BS associations and power allocations) considering the shortened distance between cellular users and BSs and the raised levels of inter-site interference.

In this thesis, we consider the unmanned aerial vehicles (UAVs) as a promising candidate to tackle these challenges in the IAB-based cellular networks. In particular, we investigate the potential gains of leveraging the flying capabilities of UAVs as hovering IAB-nodes in UAV-assisted IAB networks. There have been several recent studies where utilizing UAVs is proposed as a cost-effective and easily-scalable solution that can achieve significant performance improvements in wireless networks [8]. Specifically, the idea of using a swarm of UAVs is widely considered as a potential solution to provide wireless connectivity where users suffer from coverage gaps (see, e.g., [9] and references therein). Moreover, unlike the basic idea of dense deployment of SBSs to get closer to edge users [10], the use of UAVs allows for the network architecture to be reconfigured dynamically based on the coverage and capacity demands 11, 12.

Having UAVs communicating towards MBSs over backhaul links and towards cellular users over access links naturally leads to creating a wirelessly backhauled network architecture 13 17]. Furthermore, the entanglement between the spatial configurations of a swarm of UAVs and spatial dynamics of users' distribution makes it more challenging to meet the target quality of service (QoS) requirements. However, the joint optimization of the overall network performance in UAV-assisted IAB cellular networks considering the full and/or partial reuse of the wireless channel resources between backhaul and access links and the spatial dynamics of cellular users distribution has not been addressed in literature. Therefore, there has been 
great interest in studying the performance of UAVs on both the access and backhaul networks.

\subsection{Literature review and related work}

\section{- Applications of UAVs in cellular networks}

UAVs have been considered in literature for various applications and use cases in next-generation cellular networks. On one hand, UAVs can be integrated into cellular networks as flying BSs and can be deployed as a part of the HetNet architecture. UAVs can provide public safety communications with significant improvements in the events of small scale (e.g., building fire), and large scale (e.g., floods, hurricanes, tornadoes and military attacks) damages to the network architecture. Particularly, the throughput coverage and the fifth-percentile throughput if of the terrestrial network can be significantly improved by optimizing the 3D-deployment locations of UAVs [18]. Furthermore, it has been shown that LTE-Unlicensed (LTE-U) based UAVs can be integrated effectively with WiFi access points into UAV-assisted LTE$\mathrm{U} / \mathrm{WiFi}$ HetNet architecture to improve the coverage and capacity requirements of the terrestrial networks in the aftermath of a natural disaster [19].

UAVs can also exploit their unique characteristics (e.g. flexible, dynamic, 3D deployment and line-of-sight (LOS) communications) to improve the coverage and capacity of next-generation cellular networks. For example, AT\&T has been investigating the integration of terrestrial and traditional cell on wings (COWs) into the drones, and using them to provide additional capacity or coverage on demand. Specifically, drones can be deployed from cell towers to the desired locations (e.g., hotspots) to provide them with temporary extra capacity. UAVs can return back to the cell towers to recharge and to wait their next mission when the network deter- 
mines that the temporary capacity or coverage is no longer needed [20]. Moreover, UAVs have been exploited by Intel Corporation to create a live drone light show tethered. Drones have also been deployed above Atlanta to overlook the events surrounding the Super Bowl and add more security at the event [21].

In addition to the coverage and capacity enhancement, the unique characteristics of UAVs can be exploited to create re-configurable antenna arrays in the sky. Specifically, a group of UAVs can be spatially configured together (e.g., in the form of uniform linear array (ULA), planar array, or 3D-array) to benefit from the potential advantages of the beamforming and maximization of the antenna directivity gains 22,23$]$. In this regard, UAV-BSs are considered as promising candidates to employ massive multiple input multiple output (MIMO) and full-dimensional MIMO (i.e., 3D MIMO) in next-generation cellular networks. Generally, full-dimensional MIMO enables the 3D beamforming in both azimuth and elevation angles to maximize the received signal-to-interference-plus-noise ration (SINR) at users who are distributed at different elevation angles with respect to their serving BSs [8]. Furthermore, UAV-BSs have attracted increasing attention as a feasible, cost-effective and easily-scalable network solution that can be integrated in fast deployments of next-generation cellular networks. Specifically, the LOS capabilities of UAVs can be exploited to provide the ground networks with with reliable and cost-effective wireless backhaul connectivity $14,15,24]$.

On the other hand, UAVs can operate as cellular-connected user equipments (i.e., cellular-connected drone-UEs [25, 26]) that can be used for various applications (e.g., package delivery, internet of things (IoT) applications, remote sensing, virtual/augmented reality (VR/AR) applications and surveillance purposes). It has been shown that UAVs can be used for energy-efficient uplink data collection from ground IoT devices. Specifically, by a group of ground IoT devices can be suc- 
cessfully connected to the terrestrial network with minimum transmission power by optimizing the 3D locations of UAVs and uplink power allocations of the IoT devices. Furthermore, the connectivity of the IoT networks can be improved by intelligently moving the UAVs based on the activation patterns of the IoT devices [9]. UAVs can also be equipped with actuators, sensors, cameras and other indispensable IoT devices to enable UAVs for trajectory, path planning, environment monitoring, tracking and other purposes [27]. In [28], authors have investigated the use of facial recognition tools in a UAV-based IoT platform for crowd surveillance purposes. They have shown that how UAV can be exploited to improve the system responsiveness by quickly detecting and recognizing suspicious persons in the crowd.

In addition to the UAV-based IoT use cases, Amazon has revealed the latest version of its prime air delivery drone to improve the overall safety and efficiency of its transportation system [29]. A novel framework for the cell association and the 3D deployment of UAV-BSs and drone-UEs have proposed in 11 to improve the spectral efficiency and minimize the cell-association latency in the 3D UAV-enabled cellular networks. Furthermore, it has been found that the HetNets introduce an additional degree of freedom for UAVs to be integrated into the cellular networks as aerial-users. Specifically, UAVs at low altitudes are best served by micro cells, while UAVs at higher altitudes are best served by macro cells [30].

Although the drone-UEs leverage their LOS capabilities to establish ubiquitous and reliable wireless communication links, they might impose more interference challenges as being integrated into the cellular architecture. Generally, UAVs have a strong LOS channel to a large number of BSs, which that adding more UAVs increases the levels of UAV to ground UEs interference and UAV-UAV interference 31. Furthermore, it has been shown that adding more cellular-connected drone-UEs might decrease the network densification gains of ultra-dense networks [30]. 


\section{- Performance analysis of UAV-BS assisted cellular networks}

The access link performance gains of using UAVs as flying BSs have been studied extensively in the literature. In [9], the authors have proposed a framework to optimize the mobility and 3D deployment of UAVs to efficiently collect the uplink link data from ground internet of things (IoT) devices. It has been shown that exploiting the UAVs can significantly improve the connectivity and the energy efficiency of the IoT networks. The authors of [32] have derived the coverage probability and analyzed the performance of a communication scenario, in which, UAVs are used as hovering BSs to provide downlink link transmissions to ground users while taking into account the interference generated from the device-to-device (D2D) transmission. They have shown that the optimal UAV altitude decreases as the density of D2D users increases. Furthermore, they have proposed a framework to define the minimum number of stop points that UAV needs to coverage a desired coverage area with a minimum required transmission power. It has been shown that the number of stop points per UAVs are required to be increased to improve the coverage probability of ground users.

The interior-point optimizer of MOSEK solver and the bisection search method have been exploited in [33] to find the 3D placement that maximizes the number of covered users by a single UAV-BS. It has been demonstrated that measuring the traffic characteristics in space (e.g. the amount of clustered ground cellular users) is of significant importance for determining the efficiency of integrating the UAVs into

the cellular network architecture. It has been proved that the use of UAVs as relays in UAV-assisted HetNets can improve the capacity, reliability and connectivity of the cellular networks compared with the ground-based terrestrial networks [34]. It is also has been shown that UAVs not only provides long-range connectivity but also improve the load balancing and the traffic offload in the UAV-assisted HetNets. 
In [35], the authors have presented experimental field-test results of using small UAV (SUAV) as a wireless relay in a cellular network. They have shown that the peak throughput and ping time can be significantly improved by integrating UAVs as wireless relays into the existing cellular networks. The problem of optimizing the coverage area of a single UAV-BS has been formulated in 36 as a second order cone problem (SOCP) to find hovering altitude of the UAV that maximizes the number of covered users and minimizes the transmission power of the UAV. The author have shown that there have been significant savings in the UAV downlink transmission power and the number of covered ground users as the users get closer to each other. Interference alignment (IA) technique has been exploited in [37 to propose a blind channel state information (CSI) feedback transmission scheme that maximizes the sum-rate of a high altitude platform drone-based wireless system. It has been proven that IA can be exploited to mitigate the UAV-UAV interference and increase the sum-rate gain significantly in relay-aided drone wireless networks.

The optimal transport theory has been exploited in 38 to minimize the flight time and find the optimal deployment of UAVs to provide wireless service to ground users and minimize the transmission power of UAVs. It has been shown that the total UAV-hover time that is required for serving ground users can be decreased in increasing the allocated bandwidth to ground users (i.e., by increasing the transmission rate) [39]. The authors have also shown that the total hover time of each UAV decreases as the number of serving UAV-BSs increases given a fixed number of ground users. However, increasing the number of UAVs leads to more inter-cell interference which reduces the transmission rate gains that can be achieved by using more UAVs. It has been revealed that using UAVs with long flight time can be more beneficial than using UAVs with short flight times. The mean-field game has been exploited in [40] to propose a non-cooperative movement control algorithm for 
a swarm of massive UAVs, in which, the energy efficiency of UAVs is maximized while avoiding inter-UAV collisions. Authors have shown how the mean-field game approach can be leveraged to enable fully-distributed control of massive number of UAVs. It has also been shown how the downlink rate of ground users can be enhanced by decreasing the mechanical mobility control energy and minimizing the UAV energy consumption per downlink rate. A 3D deployment approach based on the circle packing theory has been proposed in [41] to maximize the coverage performance and minimize the transmission power of UAVs. It has been shown how the number of UAVs, the beamwidth of the antennas and the 3D locations of UAVs can be designed to meet the coverage requirements in the desired coverage area. In 42, the authors have proposed a machine learning framework to efficiently predict the congested coverage areas and subsequently deploy a group of UAV-BSs therein, to offload the traffic from congested terrestrial BSs to UAVs. It has been demonstrated that the machine learning can be used to significantly reduce the required UAV downlink transmission power and mobility power to satisfy the users' demands compared with the traditional optimal deployment of UAVs without machine learning prediction.

Millimeter-wave frequency band has been considered as a promising solution for reliable, scalable and low-latency UAV-based cellular networks for multiple reasons. First, highly directional and 3D beamforming can be exploited for interference mitigation over the backhaul and access links in UAV communications [43]. Second, the dominance of LOS links (e.g., Air-to-Ground, Ground-to-Air and Air-to-Air) suggests that mmWave frequencies can be easily exploited in UAV-assisted cellular networks [44]. Third, mmWave communication is considered as a promising candidate to establish high date rate and high capacity wireless access and backhaul connections in UAV use cases (e.g., support high traffic demands in congested ar- 
eas, data collection from ground devices and video monitoring traffic) due to the availability of large spectrum resources in mmWave frequency bands [45 48. In this regard, ray tracing simulations have been utilized in [49 to study the propagation characteristics of of outdoor mmWave channels at 30 and $60 \mathrm{GHz}$ frequency bands in UAV-assisted IAB mmWave networks. Ray tracing simulation results have shown that using UAV as amplify-and-forward (AF) and decode-and-forward (DF) relays achieves significant gains in the downlink coverage and capacity of IAB mmWave networks. An angular-based user separation approach has been proposed in [50] to find the UAV deployment that maximizes the overall downlink link rate. It has been mobility attributes of UAVs can be exploited to move the UAVs to location where orthogonal beams can be generated at different users. In that, the UAV-based beamforming scheme can give better capacity performance than that of the normal linear zero-forcing beamforming (LZFBF) without the requirement of CSI knowledge from all users in the desired coverage area (i.e., CSI is required to be known from the serving user only).

In [51], the authors have proposed distance-based CSI feedback scheme and derived the analytical expressions of outage probabilities and sum-rates for UAVbased mmWave non-orthogonal multiple access (NOMA) downlink transmissions. It has been shown that the distance-based CSI feedback can be an efficient alternative for the traditional full CSI feedback for fast varying channels in UAV-assisted mmWave networks. The authors have also demonstrated that NOMA can be exploited in UAV-based cellular networks to significantly improve the sum-rate performance compared with traditional orthogonal multiple access (OMA) schemes. UAV-BSs have been also used in [52 to design an angle-based CSI feedback scheme (by exploiting the space domain and beamforming) for mmWave NOMA downlink transmissions in UAV-assisted cellular networks. Authors have also shown how to 
determine a feedback scheme as a measure of channel quality (e.g. angle-based or distance-based) based on the UAV beamwidth, so that ground users can become more distinguishable. Furthermore, 3GPP has been investigating the integration of UAVs as hovering BSs and cellular connected drones-UEs into the existing cellular networks 26, 53.

On the backhaul network side, the authors of [14] have exploited the branchand-bound method to find deployment of a single UAV that maximizes the network utility function considering the limiting constraint of available spectrum resources for backhaul transmissions. It has been shown how the supported peak date rate on the drone access links can be limited by the capacity limitation on the wireless backhaul links. Authors also have demonstrated that the UAV deployment algorithm can be robust against the modest movement of ground users by finding the optimal 3D locations of UAVs. Hence, UAVs can be exploited to improve the coverage and capacity of the cellular network while minimizing the energy consumption of UAVs. In [15], the authors have exploited convex optimization and particle swarm optimization (PSO) algorithm to find UAV deployments and user-BS associations considering the limited available data rates at backhaul links. Authors have shown how using UAV-BSs can increase the overall network rate when users are clustered into several hotspots in the desired coverage area. It has been also shown how defining the optimal UAV-BS beamwidth can decrease the UAV-UAV interference levels in UAV-assisted cellular networks. Another PSO-based algorithm has been presented in [54], in which, different network utility functions were maximized successively, to find UAV deployments and minimum required number of UAVs to cover a designated coverage area. It has been shown how the optimal deployment of UAVBSs (i.e., number of UAV-BSs and the 3D locations of UAVs) is intertwined with the density of ground users in the desired coverage area. Specifically, a UAV-BS 
decreases its altitude in dense area to reduce the inter-cell interference and increases its altitude to increase its coverage area when the number of ground users is low (i.e., low density coverage area).

In addition to the conventional spatial configuration of UAVs as distributed nodes, the authors in [22] have exploited an evolution-based multi-objective optimization algorithm to maximize the directivity of UAV-based 3D antenna array. Specifically, they have shown how to exploit the 3D deployment attribute of UAVs as an additional degree of freedom to combine a group of UAV together and generate different antenna configurations (e.g., cubic, linear, circular, planar and 3D nonuniform antenna array). It has been demonstrated that the proposed UAVbased antenna arrays can provide significant performance improvements in terms of antenna directivity gain and side lobe level. Essentially, in UAV-based antenna configurations, UAV-BSs are not interfering to each other, but are rather benefiting from being composed in a single antenna array. The UAV-based coordinated antenna configurations help to increase the operation range of UAV-communications and improve the overall system performance without compromising the energy and weight load of UAVs. In [55], the authors have optimized the deployment of a group of single-antenna UAVs to maximize the LOS MIMO channel gain and minimize the travelling distance of each UAV. It has been shown how directing UAVs to form an optimal uniform rectangular array (URA) can be exploited to minimize the overall distance travelled by all UAVs.

The authors of [23,56] have proposed a framework that dynamically adjusts the 3D locations of drone-elements in DAA to provide wireless coverage to different ground users based on their spatial distribution. They have exploited a geometricbased solution and bang-bang control theory to minimize the transmission time and the control time that is required to adjust the 3D locations of the drones, 
respectively. It has been shown how a group of UAVs can be configured together to maximize the array directivity of the proposed drone antenna array (DAA). Authors have also demonstrated that the proposed DAA framework can significantly reduce the UAV-service time and improve the spectral and energy efficiency of the UAVassisted cellular networks. However, it is worth noting that the negative impacts of the required control time to adjust the array locations every time it serves a different user, in terms of, transmission delay and low data rates, impose various challenges on the proposed framework to be implemented in next-generation cellular networks.

\section{- Performance analysis of IAB-based cellular networks}

As mentioned earlier, wireless backhauling has been emerged as a potential solution to reduce the network deployment cost and meet the traffic demands of next-generation cellular networks. The $3 \mathrm{GPP}$ is currently considering IAB and its inherited use cases; namely out-of-band and in-band IAB; as attractive solutions to improve the spectral efficiency in wirelessly backhauled cellular networks [3,4]. The joint optimization problem of load balancing and interference mitigation in IAB 5G HetNets has been studied in [5] taking into account the dynamic wireless backhaul, traffic demands and imperfect CSI. It has been demonstrated that increasing the small cell density and improving the wireless backhaul quality can significantly improve the cell-edge performance of IAB HetNets. The power consumption of downlink and uplink transmissions in wirelessly backhauled HetNets has been studied in [57]. Authors have presented how the time-division-duplexing (TDD) can be exploited for coordination between macro and small BSs without the need of exchanging the CSI through the wireless backhaul links. It has been also shown that the network operation can become infeasible beyond a critical value of the imperfect CSI. Hence, a fraction of users are required to lower their target rates 
or to use other transmission protocols that do not require CSI knowledge at the transmitter.

Regularized zero-forcing (RZF) precoding and joint linear minimum mean square error (LMMSE) have been exploited in [58] to mitigate in inter-tier interference between uplink and downlink transmission of macro and small cells in out-of-band wirelessly backhauled cellular networks. In [6], the authors have analyzed the downlink rate coverage probability and studied different bandwidth splitting strategies between access and backhaul links in mmWave HetNets. It has been proven that, for different splitting strategies, there exists an optimal access-backhaul bandwidth split such that a specific objective function (e.g., rate coverage probability, median rate and $5^{\text {th }}$ percentile rate) can be maximized. Authors have also proved that the IAB-enabled cellular networks outperform the macro-only networks (i.e., without wireless backhauling and without SBS) up to a critical cell-load. This critical cellload is linearly proportional to the overall available system bandwidth. It is worth mentioning that the IAB-based wireless backhaul network architecture has been demonstrated to significantly improve the overall network throughput and end-toend latency in congested cellular networks [7].

In this context, the Xhaul architecture has in proposed in [59], in which, backhaul and fronthaul connections are tightly integrated together to enable flexible and software-defined reconfiguration of all networking elements in next-generation cellular networks. The joint resource allocation for D2D, access and backhaul connections has been investigated in [60] in IAB-based next generation cellular networks. The interoperability between base stations from different manufactures have been studied in 61] for multi-hop IAB-enabled cellular networks. It has been shown that the optimized relay selection and the joint resource allocation at both backhaul and access links can significantly improve the received user downlink rates. The resource 
allocation problem in mmWave self-backhauled IAB network has been studied in 62 to maximize the overall received data sum-rate at cellular users taking into account the capacity limitations at backhaul links. It has been shown how the Markov approximation can be exploited to significantly improve the spectral efficiency of the self-backhauled IAB cellular networks. Furthermore, it has been shown that the IAB network architecture can be exploited to decrease the deployment cost and improve the overall downlink and uplink sum-rates of the mmWave fixed access wireless networks 63.

\subsection{Contribution of the Thesis}

To the best of the authors' knowledge, none of the prior studies have considered in their analysis the problem of jointly optimizing the UAV deployment, user-BS associations and power allocations taking into account the tight interworking and the mutual interference between access and backhaul links, resulting inter-cell interference and the mutual dependence between the spatial configurations of the UAVs and spatial dynamics of cellular users distributions in UAV-assisted IAB networks. Moreover, none of these works considered the problem of finding the design parameters of DAA independent of the number of drone element in the DAA spatial configurations of UAVs.

In this thesis, we propose an interference management algorithm for UAV-assisted IAB cellular networks. In particular, the proposed algorithm jointly optimizes the 3D deployment of UAVs, user-BS associations and power allocations for downlink link transmissions at backhaul and access links. We show how the spatial configurations of UAVs are intertwined with the spatial distribution of ground cellular users and present two spatial configurations of UAVs; namely distributed UAVs and 
DAA; based on the spatial dynamics of ground users. Moreover, we consider inband backhauling, as a natural candidate for tighter interworking between access and backhaul links. In the former mode we define the 3D placement of UAVs. In the latter mode we define the DAA design parameters in terms of 3D placement of array center, array orientation and drone element separation. All while taking into account the mutual interference due to the full reuse of wireless channel resources, i.e., time and frequency, between backhaul and access links, LOS capabilities of UAVs, inter-cell interference and spatial dynamics of users' distribution.

The problem is cast as a network sum rate maximization problem and decomposed into two subproblems due to the mutual dependence between the optimization variables. We then propose an iterative framework to find the optimized set of variables. In that, the first subproblem is solved using a two-stage fixed-point method to find user-BS associations and downlink power allocations for access and backhaul transmissions, given fixed UAV spatial configurations. The second subproblem is solved using particle swarm optimization (PSO) to define the spatial configurations of UAVs and update power allocations given fixed user-BS associations. We show how the computational complexity of the proposed framework can be independent of the number of UAVs when they are configured as DAA, and demonstrate (with the aid of simulation results) the consistency of the computational complexity of the proposed framework for larger number of UAVs.

Our numerical results show that the proposed algorithm achieves an average of $3.1 \times$ and $6.7 \times$ gains in received downlink signal-to-interference-plus-noise ratio (SINR) and overall network sum rate, respectively, compared to the baseline scenario, in which, UAVs are not used. We demonstrate that the use of UAVs in in-band IAB networks results in both coverage enhancement and capacity boosting. As for the DAA configuration, the numerical results also reveal that the achievable 
network performance gains are directly proportional to the number of drone elements in the DAA. In this regard, we show how the computational complexity of the proposed algorithm can be independent of the number of UAVs when they are configured as DAA. We also analyze the convergence results of the proposed PSO algorithm and show how PSO settings can be adjusted to converge to the same nearoptimal set of solutions in fewer number of iterations. We discuss the robustness of the proposed iterative algorithm against the order of the optimization steps and show that it converges to same optimized set of solutions irrespective of the order of the optimization steps. Furthermore, our numerical results reveal that the performance of the proposed algorithms is directly proportional to the heterogeneity of the spatial distribution of cellular users (i.e.,performance gain increases with more clustered users).

\subsection{Organization of the Thesis}

In Chapter 2, we consider different spatial configuration modes of UAVs; namely distributed UAVs and drone antenna array; and present a system model for downlink transmissions of both access and backhaul links in UAV-assisted IAB cellular networks. Furthermore, we show how the in-band backhauling can be resulted in tighter interworking between access and backhaul links in IAB-based cellular scenarios.

In Chapter 3, we formulate the problem of joint optimization of user-BS associations, downlink power allocations and the 3D deployment of UAVs to maximize the overall network sum-rate while keeping the minimum levels of interference at access and backhaul links for different UAV configuration modes (i.e., distributed UAVs and drone antenna array) in UAV-assisted IAB cellular networks. In addition, we 
show how fixed point iteration method and PSO algorithm can be exploited to develop an iterative interference management algorithm to solve the proposed network sum-rate maximization problem.

In Chapter 4, we exploit the proposed iterative algorithm and extensive Monte Carlo simulations to numerically evaluate the performance gains of using UAVs as IAB-nodes in in-band IAB networks, in terms of the received downlink throughput, SINR at both access and backhaul links and overall network sum rate. Furthermore, we analyze the convergence results of the proposed iterative algorithm and discuss its robustness against the order of the optimization steps.

Finally, the concluding remarks are drawn in Chapter 5.

\subsection{Notation}

The following notation is used throughout the thesis. Matrices are expressed by uppercase and boldface letters. Lowercase and boldface letters represent vectors. Sets are denoted by script typefaces. We use standard normal case letters to represent the scalars. $(.)^{*},(.)^{\dagger},(.)^{\top}$ and $\oslash$ denote the Hermitian transpose, the pseudo-inverse, the transpose and the Hadamard division operations, respectively. The norm of a vector is represented by $\|$.$\| . Either the cardinality of a set or the absolute value$ of a scalar are described by $|$.$| depending on the context. We denote the complex$ Gaussian and uniform distributions by $\mathcal{C N}$ and $\mathcal{U}$, respectively. Finally, $\mathbf{1}_{A}$ denotes the $A$-dimensional all-ones vector. 
CHAPTER 2

\section{SYSTEM AND RECEIVED SIGNAL MODEL}

We consider a downlink link transmission scenario in IAB multi-tier drone cellular network as shown in Fig. 2.1. The first tier represents the IAB-donor $b$ that supports $T$ terrestrial users (tUEs) with direct links and provides wireless backhauling functionality to $D$ UAVs. The second tier represents UAVs operating as drone IAB-nodes to support $A$ aerial users (aUEs) with access links. We consider an inband -IAB scenario, in which, access and backhaul link fully overlap in spectrum resources [3]. Fig. 2.1 clarifies definitions that are used throughout this thesis to refer to the proposed system model. The downlink transmission denotes the data transmission from UAVs to aUEs, IAB-donor to tUEs, and IAB-donor to UAVs. The IAB-donor uses same spectrum resources for direct and backhaul links, and it is equipped with $N$ element uniform linear array (ULA). Drone IAB-nodes use the same spectrum resources for backhaul and access links, and they are equipped with single receiving and transmitting antennas. Similarly, cellular users are equipped with single receiving and transmitting antennas. We assume that cellular ground users are spatially distributed into $D$ clusters. Let $\mathcal{D}=\{1, \ldots, D\}, \mathcal{A}=\{1, \ldots, A\}$ and $\mathcal{T}=\{1, \ldots, T\}$ denote the sets of UAVs, aUEs and tUEs, respectively where,

e.g., the cardinality of $\mathcal{D}$ is $|\mathcal{D}|$ and is equal to $D$. The set of BSs is represented by $\mathcal{S}=\{1, \ldots, S\}$ where $S=D+1$. Finally, the set of users is represented by 


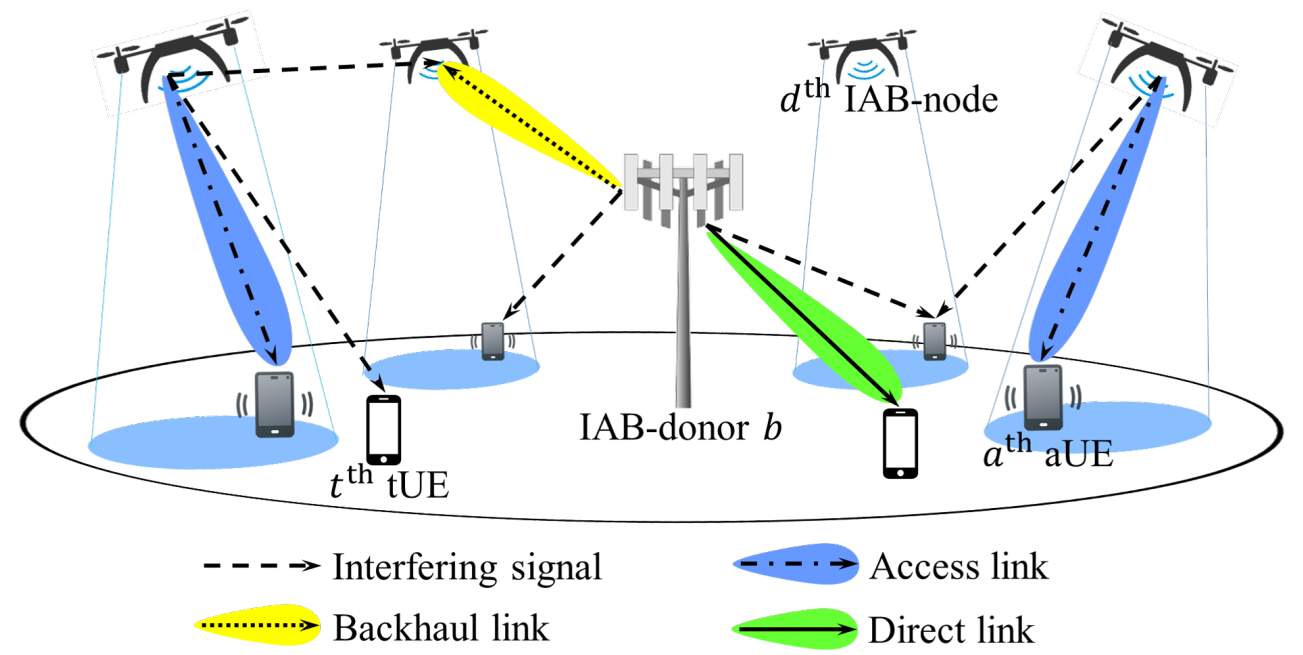

Figure 2.1: In-band IAB system architecture for next-generation cellular networks: UAVs can be users themselves or operate as drone IAB-nodes to serve other users.

$\mathcal{U}=\mathcal{A} \cup \mathcal{T}$ where $U=A+T$.

\subsection{Generic Channel Model}

The multiple-input-single-output (MISO) downlink channel $\mathbf{h}_{\mathrm{b}, t} \in \mathbb{C}^{1 \times N}$ between IAB-donor and $t^{\text {th }}$ tUE is introduced as [64, Ch. 7]:

$$
\mathbf{h}_{\mathrm{b}, t}=\frac{1}{\sqrt{K}} \times \sum_{k=1}^{K} \frac{g_{\mathrm{bt}, k} \mathbf{a}^{*}\left(\theta_{\mathrm{b} t, k}\right)}{1+\left(d_{\mathrm{b}, t}\right)^{\alpha}},
$$

where $K, g_{\mathrm{bt}, k}, \theta_{\mathrm{bt}, k}, d_{\mathrm{b}, t}$ and $\alpha$ represent the number of propagation paths, complex channel gain of the $k^{\text {th }}$ path, angle-of-departure (AoD) of the $k^{\text {th }}$ path, 3D distance between IAB-donor and $t^{\text {th }}$ tUE and pathloss coefficient, respectively. $g_{\mathrm{b} t, k}$ follows standard complex Gaussian distribution with $\mathcal{C N}(0,1)$ and $\theta_{\mathrm{b} t, k}$ follows a uniform distribution with $\mathcal{U}\left[\theta_{\mathrm{b}, t}^{\mathrm{LOS}}-\mathrm{ASD}, \theta_{\mathrm{b}, t}^{\mathrm{LOS}}+\mathrm{ASD}\right]$ where $\theta_{\mathrm{b}, t}^{\mathrm{LOS}}$ is the LOS angle between IAB-donor and $t^{\text {th }}$ tUE, and ASD is the angular spread of departure and follows the same distribution as [65, Table 7.5-6]. The transmit antenna array steering vector 
of the $k^{\text {th }}$ path and AoD $\theta_{\mathrm{bt}, k}$ is given by:

$$
\mathbf{a}\left(\theta_{\mathrm{b} t, k}\right)=\frac{1}{\sqrt{N}}\left[1, e^{-j 2 \pi \frac{\Delta}{\lambda} \sin \left(\theta_{\mathrm{b} t, k}\right)}, \ldots, e^{-j 2 \pi \frac{\Delta}{\lambda}(N-1) \sin \left(\theta_{\mathrm{b} t, k}\right)}\right]^{\top}
$$

where $\Delta$ is the antenna element separation of the ULA and $\lambda$ is the carrier wavelength. Similarly, the backhaul channel between IAB-donor and $d^{\text {th }}$ drone is represented by $\mathbf{h}_{\mathrm{b}, d} \in \mathbb{C}^{1 \times N}$ and the access channel between $d^{\text {th }}$ drone and $a^{\text {th }}$ aUE is represented by $h_{d, a} \in \mathbb{C}^{1 \times 1}$.

\subsection{Distributed UAVs Spatial Configuration Mode}

\subsubsection{Backhaul Downlink Transmissions}

We consider linear zero-forcing beamforming (LZFBF) for multi-user MISO transmissions at backhaul links, in which, the ZF precoder at IAB-donor is defined as $\mathbf{V}_{\mathrm{b}} \in \mathbb{C}^{N \times(D+1)}$, where $\mathbf{V}_{\mathrm{b}}=\mathbf{H}_{\mathrm{b}}^{\dagger}=\mathbf{H}_{\mathrm{b}}^{*}\left[\mathbf{H}_{\mathrm{b}} \mathbf{H}_{\mathrm{b}}^{*}\right]^{-1}$. The full rank channel matrix between IAB-donor, UAVs and $t^{\text {th }}$ tUE scheduled at $f^{\text {th }}$ subcarrier and $l^{\text {th }}$ time slot is given by $\mathbf{H}_{\mathrm{b}}(f, l) \in \mathbb{C}^{(D+1) \times N}$ where $\mathbf{H}_{\mathrm{b}}(f, l)=\left[\mathbf{h}_{\mathrm{b}, 1}(f, l), \ldots, \mathbf{h}_{\mathrm{b}, D}(f, l), \mathbf{h}_{\mathrm{b}, t}(f, l)\right]$. For simplicity of presentation, we omit references to $(f, l)$ indices in the rest of this thesis. The precoding vector between IAB-donor and $i^{\text {th }}$ reception point is normalized using equal transmit power (ETP) normalization due to its higher sum rate gains $\left[66\right.$, and is given by $\mathbf{v}_{\mathrm{b}, i}=\left[\mathbf{V}_{\mathrm{b}}\right]_{i} /\left\|\left[\mathbf{V}_{\mathrm{b}}\right]_{i}\right\|$, where $\left[\mathbf{V}_{\mathrm{b}}\right]_{i}$ is the $i^{\text {th }}$ column of $\mathbf{V}_{\mathrm{b}}$.

The received signal at $d^{\text {th }}$ drone from IAB-donor (see Fig. 2.1) can be modeled 
as:

$$
\begin{aligned}
y_{\mathrm{b}, d} & =\underbrace{\sqrt{p_{\mathrm{b}, d}} \mathbf{h}_{\mathrm{b}, d} \mathbf{v}_{\mathrm{b}, d} x_{\mathrm{b}, d}}_{\text {transmitted signal }}+\underbrace{\sum_{i \in \overline{\mathcal{A}}_{d}} \sqrt{p_{d, i}} h_{d, d} x_{d, i}}_{\text {self-interference }}+\underbrace{\sum_{i \in \overline{\mathcal{A}}_{\mathrm{b}}} \sqrt{p_{\mathrm{b}, i}} h_{\mathrm{b}, d} \mathbf{v}_{\mathrm{b}, i} x_{\mathrm{b}, i}}_{\text {inter-stream interference }} \\
& +\underbrace{\sum_{j \in \mathcal{D} \backslash d} \sum_{i \in \overline{\mathcal{A}}_{j}} \sqrt{p_{j, i}} h_{j, d} x_{j, i}+n_{d},}_{\text {inter-tier interference }}
\end{aligned}
$$

where $p_{\mathrm{b}, d}, \mathbf{v}_{\mathrm{b}, d}$ and $x_{\mathrm{b}, d}$ represent the backhaul downlink power allocation, precoding vector and transmitted data symbol. $\overline{\mathcal{A}}_{d}$ and $\overline{\mathcal{A}}_{j}$ denote the sets of interfering aUEs that are associated with $d^{\text {th }}$ and $j^{\text {th }}$ UAVs, respectively where $j \neq d$. The second, third and fourth terms in (2.3) represent the self-interference between access and backhaul, inter-stream interference and inter-tier interference on backhaul transmissions of $d^{\text {th }}$ drone. $n_{d} \sim \mathcal{C N}\left(0, \sigma^{2}\right)$ denotes the received zero-mean complex Gaussian noise with variance $\sigma^{2}$ at $d^{\text {th }}$ UAV. Each UAV is a full-duplex capable drone IAB-node, which can be integrated into in-band IAB scenarios without self-interference constraints. We assume perfect channel state information (CSI) knowledge at IAB-donor. Further, LZFBF is used to suppress the inter-stream interference between $(D+1)$ independent spatial streams of backhaul and direct links [67]. Hence, the second and third terms can be omitted from (2.3) and the received SINR at $d^{\text {th }}$ drone can be calculated as:

$$
\gamma_{\mathrm{b}, d}=\frac{p_{\mathrm{b}, d}\left|\mathbf{h}_{\mathrm{b}, d} \mathbf{v}_{\mathrm{b}, d}\right|^{2}}{\sum_{j \in \mathcal{D} \backslash d}\left|h_{j, d}\right|^{2} \sum_{i \in \mathcal{A}_{j}} p_{j, i}+\sigma^{2}} .
$$

\subsubsection{Access Downlink Transmissions}

Similarly, the received downlink signal at $t^{\text {th }}$ tUE from IAB-donor is given by:

$$
y_{\mathrm{b}, t}=\underbrace{\sqrt{p_{\mathrm{b}, t}} \mathbf{h}_{\mathrm{b}, t} \mathbf{v}_{\mathrm{b}, t} x_{\mathrm{b}, t}}_{\text {transmitted signal }}+\underbrace{\sum_{j \in \mathcal{D}} \sum_{i \in \overline{\mathcal{A}}_{j}} \sqrt{p_{j, i}} h_{j, t} x_{j, i}}_{\text {inter-tier interference }}+n_{t},
$$


where $\overline{\mathcal{A}}_{j}$ is the set of associated aUEs with $j^{\text {th }}$ drone and are scheduled on same spectrum and time resources as $t^{\text {th }}$ tUE. The second term in (2.5) represents the inter-tier interference on the access transmissions of $t^{\text {th }}$ tUE. The received SINR at $t^{\text {th }}$ tUE can be expressed by:

$$
\gamma_{\mathrm{b}, t}=\frac{p_{\mathrm{b}, t}\left|\mathbf{h}_{\mathrm{b}, t} \mathbf{v}_{\mathrm{b}, t}\right|^{2}}{\sum_{j \in \mathcal{D}}\left|h_{j, t}\right|^{2} \sum_{i \in \overline{\mathcal{A}}_{j}} p_{j, i}+\sigma^{2}} .
$$

Finally, the received downlink signal at $a^{\text {th }}$ aUE from $d^{\text {th }}$ drone is given by:

$$
y_{d, a}=\underbrace{\sqrt{p_{d, a}} h_{d, a} x_{d, a}}_{\text {transmitted signal }}+\underbrace{\sum_{j \in \mathcal{D} \backslash d} \sum_{i \in \overline{\mathcal{A}}_{j}} \sqrt{p_{j, i}} h_{j, a} x_{j, i}}_{\text {intra-tier interference }}+\underbrace{\sum_{k \in \mathcal{D} \cup \overline{\mathcal{T}}} \sqrt{p_{\mathrm{b}, k}} \mathbf{h}_{\mathrm{b}, a} \mathbf{v}_{\mathrm{b}, k} x_{\mathrm{b}, k}}_{\text {inter-tier interference }}+n_{a},
$$

where $\mathcal{D} \cup \overline{\mathcal{T}}$ is the set of UAVs and tUEs scheduled on the same spectrum and time resources as $a^{\text {th }}$ aUE. The second and third terms in 2.7) represent the intra-tier interference and inter-tier interference of the IAB-donor transmissions on the access transmissions of $a^{\text {th }}$ aUE. The received SINR at $a^{\text {th }}$ aUE is represented by:

$$
\gamma_{d, a}=\frac{p_{d, a}\left|h_{d, a}\right|^{2}}{\sum_{j \in \mathcal{D} \backslash d}\left|h_{j, a}\right|^{2} \sum_{i \in \overline{\mathcal{A}}_{j}} p_{j, i}+\sum_{k \in \mathcal{D} \cup \overline{\mathcal{T}}} p_{\mathrm{b}, k}\left|\mathbf{h}_{\mathrm{b}, a} \mathbf{v}_{\mathrm{b}, k}\right|^{2}+\sigma^{2}} .
$$

\subsection{Drone Antenna Array Spatial Configuration Mode}

In the previous sections, we presented how a group of UAVs can be spatially configured as distributed IAB-nodes to serve multiple hotspots for in-band IAB scenarios. As the number of ground users increases, the number of required UAVs for coverage enhancement and capacity boosting increases as well, entailing more design challenges and higher levels of interference between direct, access and backhaul links. Moreover, the SINR formulas in (2.4), (2.6) and (2.8) show that decreasing the intersite distance poses more technical challenges in the design of the proposed in-band 


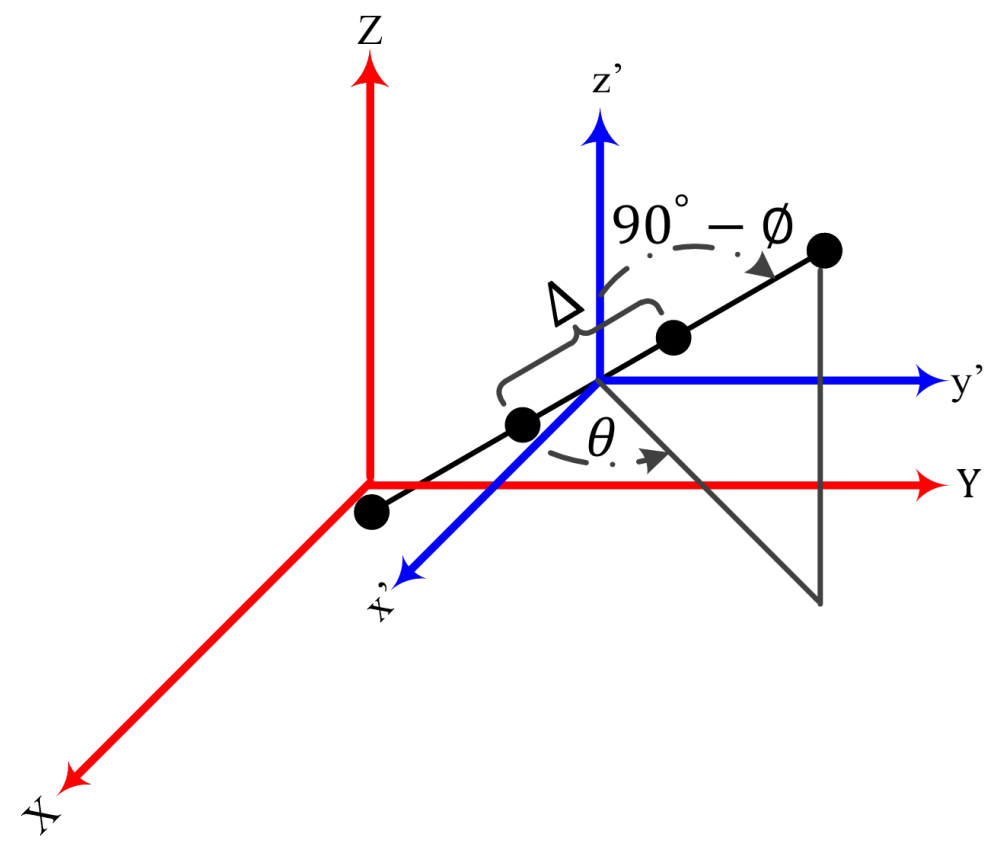

Figure 2.2: Drone antenna array design parameters.

IAB drone network architecture. To this end, we consider another spatial configuration mode for UAVs. In that, UAVs are configured as a single DAA to serve ground users that are spatially distributed in a single hotspot. Unlike distributed UAVs, UAVs in DAA mode are not interfering to each other, but are rather composed in a single antenna array to benefit from the potential advantages of the DAA [23]. The DAA configuration mode allows for on-demand array configurations. Specifically, the design parameters of the DAA are adjusted based on the spatial distribution of ground users to maximize the overall sum rate gains. 


\subsubsection{Backhaul Downlink Transmissions}

The MISO channel between DAA r, composed of single antenna $D$ drones and $a^{\text {th }}$ aUE $\mathbf{h}_{\mathrm{r}, a} \in \mathbb{C}^{(1 \times D)}$ is given by:

$$
\mathbf{h}_{\mathrm{r}, a}=\frac{1}{\sqrt{D}} \times\left[h_{1, a}, \ldots, h_{D, a}\right]
$$

where $h_{d, a} \in \mathbb{C}^{(1 \times 1)}$ is the access link channel coefficient between $d^{\text {th }}$ antenna, i.e., drone, element and $a^{\text {th }}$ aUE. It follows the same definition as 2.1). Let us consider the set of DAA design parameters as $\mathcal{X}$ where $\mathcal{X}=\left\{\theta, \phi, \Delta_{\mathrm{r}}, x_{c}, y_{c}, z_{c}\right\} . \theta \in[0,2 \pi]$, $\phi \in[0,2 \pi], \Delta_{\mathrm{r}}$ and $\left[x_{c}, y_{c}, z_{c}\right]$ are the azimuth angle from $\left(x^{\prime}, z^{\prime}\right)$ plane, elevation angle from $\left(x^{\prime}, y^{\prime}\right)$ plane, antenna element separation and 3D coordinates of the DAA center, i.e., coordinates of the origin of $\left(x^{\prime}, y^{\prime}, z^{\prime}\right)$ plane, respectively (see Fig. 2.2. Now the 3D coordinates of $d^{\text {th }}$ drone element in the DAA can be defined in terms of $\mathcal{X}$ and they are given by:

$$
\left[x_{d}, y_{d}, z_{d}\right]=\left[x_{c}, y_{c}, z_{c}\right]+\frac{\Delta_{\mathrm{r}}(D-2 d+1)}{2} \times[\cos (\phi) \cos (\theta), \cos (\phi) \sin (\theta), \sin (\phi) \sin (\theta)] .
$$

Consequently, $\mathbf{h}_{\mathrm{r}, a}$ can be defined in terms of $\mathcal{X}$ as $\mathbf{h}_{\mathrm{r}, a}(\mathcal{X})$ and is used to define LZFBF precoder for multi-user MISO transmissions at access links of the DAA. The LZFBF precoder at the DAA is given by $\mathbf{V}_{\mathrm{r}} \in \mathbb{C}^{(D \times L)}=\mathbf{H}_{\mathrm{r}}^{\dagger}=\mathbf{H}_{\mathrm{r}}^{*}\left[\mathbf{H}_{\mathrm{r}} \mathbf{H}_{\mathrm{r}}^{*}\right]^{-1}$, where $\mathbf{H}_{\mathrm{r}} \in \mathbb{C}^{(L \times D)}$ is the full rank channel matrix between DAA and $L$ aUEs with $\mathbf{H}_{\mathrm{r}}(\mathcal{X})=\left[\mathbf{h}_{\mathrm{r}, 1}(\mathcal{X}), \ldots, \mathbf{h}_{\mathrm{r}, L}(\mathcal{X})\right]^{\top}$. By utilizing the DAA configuration mode, DAA divides aUEs into spatial division multiple access (SDMA) groups. In that, the set of SDMA group of aUEs that are associated with the DAA and scheduled at same time and spectrum resources is represented by $\mathcal{L}$ where $|\mathcal{L}|=L$. It is worth noting that, the spatial multiplexing gains are constrained by the number of drones in DAA. In particular, the DAA exploits LZFBF to transmit $L$ independent spatial streams for downlink access transmissions, where $L \leq D$. 
Now, let us consider DAA that is configured to serve a group of ground users that are spatially distributed away from IAB-donor and concentrated in the center of a single hotspot. Hence, the received signal at $d^{\text {th }}$ antenna element from IAB-donor can be written as:

$$
y_{\mathrm{b}, d}=\underbrace{\sqrt{p_{\mathrm{b}, d}} \mathbf{h}_{\mathrm{b}, d} \mathbf{v}_{\mathrm{b}, d} x_{\mathrm{b}, d}}_{\text {transmitted signal }}+\underbrace{\sum_{j \in \mathcal{D} \backslash d} \sum_{i \in \mathcal{L}} \sqrt{p_{\mathrm{r}, i}} \mathbf{h}_{j, d} \mathbf{v}_{\mathrm{r}, i} x_{\mathrm{r}, i}}_{\text {self-interference }}+\underbrace{\sum_{j \in \mathcal{D} \backslash d} \sqrt{p_{\mathrm{b}, j}} \mathbf{h}_{\mathrm{b}, d} \mathbf{v}_{\mathrm{b}, j} x_{\mathrm{b}, j}}_{\text {inter-stream interference }}+n_{d},
$$

where $\mathcal{L}$ is the SDMA group of interfering aUEs to $t^{\text {th }}$ tUE . The second and third term in 2.11) denote the self-interference and inter-stream interference on the backhaul transmissions of the DAA. The received SINR at $d^{\text {th }}$ drone can be defined as:

$$
\gamma_{\mathrm{b}, d}=\frac{p_{\mathrm{b}, d}\left|\mathbf{h}_{\mathrm{b}, d} \mathbf{v}_{\mathrm{b}, d}\right|^{2}}{\sigma^{2}}
$$

\subsubsection{Access Downlink Transmissions}

Similarly, the received downlink signal and SINR at $t^{\text {th }}$ tUE from IAB-donor are given by:

$$
\begin{gathered}
y_{\mathrm{b}, t}=\underbrace{\sqrt{p_{\mathrm{b}, t}} \mathbf{h}_{\mathrm{b}, t} \mathbf{v}_{\mathrm{b}, t} x_{\mathrm{b}, t}}_{\text {transmitted signal }}+\underbrace{\sum_{i \in \mathcal{L}} \sqrt{p_{\mathrm{r}, i}} \mathbf{h}_{\mathrm{r}, t} \mathbf{v}_{\mathrm{r}, i} x_{\mathrm{r}, i}}_{\text {inter-tier interference }}+n_{t}, \\
\gamma_{\mathrm{b}, t}=\frac{p_{b, t}\left|\mathbf{h}_{\mathrm{b}, t} \mathbf{v}_{\mathrm{b}, t}\right|^{2}}{\sum_{i \in \mathcal{L}} p_{\mathrm{r}, i}\left|\mathbf{h}_{\mathrm{r}, t} \mathbf{v}_{\mathrm{r}, i}\right|^{2}+\sigma^{2}},
\end{gathered}
$$

respectively, where $\mathcal{D} \cup \overline{\mathcal{T}}$ denotes the set of interfering direct and backhaul link transmissions to $a^{\text {th }}$ UE and make interference. Finally, the received downlink signal and SINR at $a^{\text {th }}$ aUE from DAA are defined as 2.15) and 2.16, respectively where:

$$
y_{\mathrm{r}, a}=\underbrace{\sqrt{p_{\mathrm{r}, a}} \mathbf{h}_{\mathrm{r}, a} \mathbf{v}_{\mathrm{r}, a} x_{\mathrm{r}, a}}_{\text {transmitted signal }}+\underbrace{\sum_{k \in \mathcal{D} \cup \overline{\mathcal{T}}} \sqrt{p_{\mathrm{b}, k}} \mathbf{h}_{\mathrm{b}, a} \mathbf{v}_{\mathrm{b}, k} x_{\mathrm{b}, k}}_{\text {inter-tier interference }}+n_{a},
$$




$$
\gamma_{\mathrm{r}, a}=\frac{p_{\mathrm{r}, a}\left|\mathbf{h}_{\mathrm{r}, a} \mathbf{v}_{\mathrm{r}, a}\right|^{2}}{\sum_{k \in \mathcal{D} \cup \overline{\mathcal{T}}} p_{\mathrm{b}, k}\left|\mathbf{h}_{\mathrm{b}, a} \mathbf{v}_{\mathrm{b}, k}\right|^{2}+\sigma^{2}}
$$




\section{CHAPTER 3}

\section{SUM-RATE MAXIMIZATION PROBLEM IN UAV-ASSISTED IAB CELLULAR NETWORKS}

\subsection{Distributed UAVs Spatial Configuration Mode}

In this chapter, we formulate the joint optimization of user-BS associations, downlink power allocations and the 3D deployment of UAVs. To this end, The problem is cast as a network sum rate maximization problem subject to a received SINR threshold at each reception point and taking into account the transmission power constraint at each BS. The network sum rate maximization problem can be written as:

$$
\begin{array}{ll}
\mathcal{P}: \max _{\mathbf{C}, \mathbf{w}, \mathbf{p}, \mathbf{p}_{\mathrm{BH}}} & \mathbf{1}_{A}^{\top} \log _{2}\left(1+\boldsymbol{\gamma}_{\mathcal{A}}\right)+\mathbf{1}_{T}^{\top} \log _{2}\left(1+\boldsymbol{\gamma}_{\mathcal{T}}\right), \\
\text { subject to } & \boldsymbol{\gamma}_{\mathcal{U}} \geq \epsilon_{\mathrm{u}}, \boldsymbol{\gamma}_{\mathcal{D}} \geq \epsilon_{\mathrm{d}}, \\
& c_{d} \in\left[c_{d}^{(\min )}, c_{d}^{(\max )}\right], \forall c \in\{x, y, z\} \\
& \mathbf{m} \leq \mathbf{p}_{\mathcal{S}}^{(\max )}
\end{array}
$$

where $\mathbf{1}_{A}$ denotes the $A$-dimensional all-ones vector, $\gamma_{\mathcal{A}}=\left(\gamma_{a}: a \in \mathcal{A}\right)$ and $\boldsymbol{\gamma}_{\mathcal{T}}=$ $\left(\gamma_{t}: t \in \mathcal{T}\right)$ denote the vectors of received downlink SINR at aUEs and tUEs, respectively. $\mathbf{C} \in \mathbb{R}^{3 \times D}$ denotes the $3 \mathrm{D}$ locations of UAVs with $\mathbf{c}_{d}=\left[x_{d}, y_{d}, z_{d}\right]^{\top}$. The user-BS association vector is given by $\mathbf{w} \in \mathbb{R}^{1 \times U}$ where $\mathbf{w}=\left(w_{s, u}: u \in \mathcal{U}\right)$ contains the indices of serving BS of each user with value $w_{s, u}:=s, s \in \mathcal{S}$. The user

power allocation vector is given by $\mathbf{p} \in \mathbb{R}^{1 \times U}$, where $\mathbf{p}=\left(p_{s, u}: u \in \mathcal{U}\right)$ with $p_{s, u}$ being the power allocated by $s^{\text {th }}$ BS for downlink transmissions of $u^{\text {th }}$ user based on association vector $\mathbf{w}$. Similarly, the UAV backhaul link power allocation vector is given by $\mathbf{p}_{\mathrm{BH}} \in \mathbb{R}^{1 \times D}$, where $\mathbf{p}_{\mathrm{BH}}=\left(p_{b, d}: d \in \mathcal{D}\right)$. 
Essentially, a low-quality backhaul link will bottleneck the access link. In this thesis, we implement such dependency between the backhaul and access links in a binary fashion, as shown in the inequality constraint (3.2a). In that, there will be no access transmissions if the received SINR levels at backhaul links are below a predefined threshold. It is worth noting that this binary dependency resembles selective decode-and-forward (DF) relaying mode, in which, the relay only forwards the signal if the received SINR exceeds a given threshold [68]. Finally, the boundaries of the feasible set of solutions are given by $(3.2 \mathrm{~b})$ and $(3.2 \mathrm{c})$. In that, the total power allocation vector of BSs is represented by $\mathbf{m} \in \mathbb{R}^{1 \times S}$ with $\mathbf{m}=\left(m_{s}: s \in \mathcal{S}, m_{s}=\mathbf{1}^{\top} \mathbf{p}_{s}\right)$ where $\mathbf{p}_{s}=\left(p_{s, i}: i \in \mathcal{A}_{s}\right)$ and $\mathcal{A}_{s}$ denote the power allocation vector and the total number of attached users to $s^{\text {th }}$ BS, respectively. The transmission power constraints of BSs are given by $\mathbf{p}_{\mathcal{S}}^{(\max )}=\left(p_{s}^{(\max )}: s \in \mathcal{S}\right)$.

According to the channel model in (2.1), logarithmic objective function in (3.1) and SINR constraints in (3.2a), the problem is considered as NP-hard mixed-integer nonlinear program (NP-MINLP) [69]. Moreover, the problem cannot be considered as a single optimization problem due to the mutual dependence between the optimization variables. Essentially, increasing the downlink power allocations increases the received levels of signal power at cellular users and UAVs. However, given (2.4), 2.6) and (2.8), the received levels of inter-tier and intra-tier interference increase as we increase the downlink power allocations. We also note that each suboptimal set of 3D locations of UAVs leads to different suboptimal sets of userBS associations and power allocations. Hence, we solve the master optimization problem (3.1) to find the near-optimal set of power allocations, user-BS associations and 3D deployment of UAVs to maximize the received overall network downlink throughput while keeping the minimum levels of interference at access and backhaul links. 
To this end, and to make the optimization problem tractable, we decompose the mater problem in (3.1) into two subproblems denoted by $\mathcal{P} \mathcal{A}$ and $\mathcal{P B}$. In $\mathcal{P} \mathcal{A}$, we jointly optimize the user-BS associations and power allocations for access and backhaul downlink transmissions given fixed UAV spatial configurations. $\mathcal{P} \mathcal{A}$ can be written as follows:

$$
\begin{aligned}
\mathcal{P A} & : \max _{\mathbf{w}, \mathbf{p}, \mathbf{p}_{\mathrm{BH}}} \mathbf{1}_{A}^{\top} \log _{2}\left(1+\boldsymbol{\gamma}_{\mathcal{A}}\right)+\mathbf{1}_{T}^{\top} \log _{2}\left(1+\boldsymbol{\gamma}_{\mathcal{T}}\right), \\
& \text { subject to } 3.2 \mathrm{a} \text { and } 3.2 \mathrm{c})
\end{aligned}
$$

In $\mathcal{P B}$, we define the 3D hovering locations of UAVs and update downlink power allocations accordingly, given fixed user-BS associations. The subproblem $\mathcal{P B}$ is given by:

$$
\begin{aligned}
\mathcal{P B}: \max _{\mathbf{C}, \mathbf{p}, \mathbf{p}_{\mathrm{BH}}} \mathbf{1}_{A}^{\top} \log _{2}\left(1+\boldsymbol{\gamma}_{\mathcal{A}}\right)+\mathbf{1}_{T}^{\top} \log _{2}\left(1+\boldsymbol{\gamma}_{\mathcal{T}}\right), \\
\text { subject to } 3.2 \mathrm{a}-3.2 \mathrm{c} .
\end{aligned}
$$

\subsection{Drone Antenna Array Spatial Configuration Mode}

Now, we show how the network performance can be improved in in-band IAB scenarios by spatially configuring UAVs as a single DAA. The network sum rate maximization problem is given by:

$$
\begin{array}{ll}
\max _{\mathcal{X}, \mathbf{w}, \mathbf{p}, \mathbf{p}_{\mathrm{BH}}} & \mathbf{1}_{A}^{\top} \log _{2}\left(1+\boldsymbol{\gamma}_{\mathcal{A}}\right)+\mathbf{1}_{T}^{\top} \log _{2}\left(1+\boldsymbol{\gamma}_{\mathcal{T}}\right), \\
\text { subject to } & \boldsymbol{\gamma}_{\mathfrak{u}} \geq \epsilon_{\mathrm{u}}, \boldsymbol{\gamma}_{\mathcal{D}} \geq \epsilon_{\mathrm{d}} \\
& \left|\Delta_{d+1}^{(\mathrm{r})}-\Delta_{d}^{(\mathrm{r})}\right| \geq \Delta_{\mathrm{r}}^{(\min )}, \forall d \in \mathcal{D}, \\
& \theta \in[0,2 \pi[, \phi \in[0,2 \pi[ \\
& \mathbf{m} \leq \mathbf{p}_{\mathcal{S}}^{(\max )}
\end{array}
$$


where the minimum separation between the DAA antenna elements is defined in $3.6 \mathrm{~b}$ as $\Delta_{\mathrm{r}}^{(\min )}$ to avoid collisions. As shown in 3.5 , the problem is cast in terms of $\mathcal{X}$ and is independent of the number of antenna elements of the DAA. In DAA-assisted in-band IAB scenarios, the network performance enhancement is directly proportional to the number of antenna elements of the DAA (see Section 4.1). Hence, it is of paramount importance to design problem (3.5) such that its computational complexity is independent of the number of UAVs. Problem s.5 shares the same logarithmic objective function and SINR non-linear inequality constraints as 3.1. Hence, both problems can be solved using the two-stage iterative algorithm in Algorithm 3 as will be shown in the following sections. Finally, $\mathcal{P} \mathcal{A}$ and $\mathcal{P B}$ can be defined as (3.7) and (3.8), respectively where:

$$
\begin{aligned}
& \mathcal{P} \mathcal{A}: \max _{\mathbf{w}, \mathbf{p}, \mathbf{p}_{\mathrm{BH}}} \mathbf{1}_{A}^{\top} \log _{2}\left(1+\boldsymbol{\gamma}_{\mathcal{A}}\right)+\mathbf{1}_{T}^{\top} \log _{2}\left(1+\boldsymbol{\gamma}_{\mathcal{T}}\right), \\
&\text { subject to } 3.6 \mathrm{a} \text { and } 3.6 \mathrm{~d}), \\
& \mathcal{P B}: \max _{\mathcal{X}, \mathbf{p}, \mathbf{p}_{\mathrm{BH}}} \mathbf{1}_{A}^{\top} \log _{2}\left(1+\boldsymbol{\gamma}_{\mathcal{A}}\right)+\mathbf{1}_{T}^{\top} \log _{2}\left(1+\boldsymbol{\gamma}_{\mathcal{T}}\right), \\
& \\
& \text { subject to } 3.6 \mathrm{a}-3.6 \mathrm{~d} .
\end{aligned}
$$

\subsection{Hybrid Fixed-Point Iteration and Particle Swarm Ap- proach}

First we exploit fixed-point method and PSO to solve $\mathcal{P} \mathcal{A}$ and $\mathcal{P B}$, respectively. An iterative algorithm is then presented to jointly optimize user-BS associations, power allocations and the $3 \mathrm{D}$ locations of $\mathrm{UAV}$ s by exploiting $\mathcal{P} \mathcal{A}$ and $\mathcal{P} \mathcal{B}$. The proposed algorithm converges to a near-optimal feasible set of solutions after a finite number of iterations. The optimization variables are updated every update time instant the 
network reaches a predefined user-drop rate, or when the quality of service (QoS) of a certain group of users decreases below a predetermined level.

\subsubsection{Fixed-point Iteration Method}

Let us first consider uniform random initialization for user-BS associations, where $\mathbf{w}(0) \sim \mathcal{U}[1, D]$. Similarly, the UAV 3D location matrix is initialized with uni-

formly distributed random locations between $c_{d}^{(\min )}$ and,$c_{d}^{(\max )}$, where $\mathbf{C}(0) \sim$ $\mathcal{U}\left[c_{d}^{(\min )}, c_{d}^{(\max )}\right]$. The downlink access and backhaul power allocations are also initialized with equal allocations based on the number of associated users with each BS, where $p_{s, u}:=p_{s}^{(\max )} / \mathcal{A}_{s}$ and $p_{\mathrm{b}, d}:=p_{\mathrm{b}}^{(\max )} / \mathcal{A}_{\mathrm{b}}$. Now, let $t_{s, u}$ be the required power to receive unity SINR when $u^{\text {th }}$ user is associated with $s^{\text {th }}$ BS. In other words, given 2.6 it can be calculated at $t^{\text {th }}$ tUE as:

$$
t_{\mathrm{b}, t}=\frac{\sum_{j \in \mathcal{D}}\left|h_{j, t}\right|^{2} \sum_{i \in \overline{\mathcal{A}}_{j}} p_{j, i}+\sigma^{2}}{\left|\mathbf{h}_{\mathrm{b}, t} \mathbf{v}_{\mathrm{b}, t}\right|^{2}} .
$$

Hence, the matrix of required power allocations to have a unity SINR at all users can be written as $\mathbf{T}_{\mathrm{u}} \in \mathbb{R}^{S \times U}$, where $t_{s, u}$ denotes the value of element $\mathbf{T}_{\mathrm{u}}[s, u]$. In other words, $\mathbf{T}_{\mathrm{u}}$ calculates the required power allocation at $u^{\text {th }}$ user to receive a unity SINR when it is associated with $s^{\text {th }}$ BS $\forall s \in \mathcal{S}$. The optimum power allocation at each user is defined as the minimum power allocation among all BSs. Hence, the user-power allocation vector of $(i+1)^{\text {th }}$ iteration can be updated as:

$$
\mathbf{p}(i+1) \leftarrow \min _{s \in \mathcal{S}} t_{s, u}(i), u \in \mathcal{U}
$$

where $\mathbf{p}$ is a vector of column-minima of $\mathbf{T}_{\mathrm{u}}$. The corresponding user-BS association can be given accordingly by:

$$
\mathbf{v}(i+1) \leftarrow \underset{s \in \mathcal{S}}{\arg \min } t_{s, u}(i), u \in \mathcal{U}
$$


Similarly, the required backhaul power allocations to receive unity SINR at UAVs is denoted by $\mathbf{t}_{\mathrm{BH}}(i+1) \in \mathbb{R}^{1 \times D}$ and is computed based on the association vector $\mathbf{v}(i+1)$.

Now, for $t^{\text {th }}$ tUE to receive a minimum SINR of $\epsilon_{\mathrm{u}}$, the user power allocation in (3.9) can be updated as $t_{\mathrm{b}, t} \leftarrow \epsilon_{\mathrm{u}} t_{\mathrm{b}, t}$. In other words, if a power allocation of $t_{\mathrm{b}, t}$ gives an $\operatorname{SINR}=1$, then a power allocation of $\epsilon_{\mathrm{u}} t_{\mathrm{b}, t}$ gives an $\operatorname{SINR}=\epsilon_{\mathrm{u}}$. Given that $\mathbf{p}(i+1) \in \mathbb{R}^{1 \times U}$ in (14) denotes the optimum user-power allocation vector of $(i+1)^{\text {th }}$ iteration to reach a unity SINR at each user, it can be updated as follows:

$$
\mathbf{p}(i+1) \leftarrow \epsilon_{\mathrm{u}} \mathbf{p}(i+1)
$$

in order to receive a minimum SINR of $\epsilon_{\mathrm{u}}$ at all users. Similarly, given that $\mathbf{t}_{\mathrm{BH}}(i+1) \in \mathbb{R}^{1 \times D}$ is the optimum backhaul power allocations of $(i+1)^{\text {th }}$ iteration to receive unity SINR at UAVs, the backhaul power allocation vector $\mathbf{p}_{\mathrm{BH}} \in \mathbb{R}^{1 \times D}$ can updated as follows:

$$
\mathbf{p}_{\mathrm{BH}}(i+1) \leftarrow \epsilon_{\mathrm{d}} \mathbf{t}_{\mathrm{BH}}(i+1),
$$

in order to receive a minimum SINR of $\epsilon_{\mathrm{d}}$ at all UAVs. For simple notations, we omit references to index $i$ throughout the rest of this chapter.

Next, we adjust the updated user and backhaul power allocations based on the total power allocations of each BS to satisfy the inequality constraint in $3.2 \mathrm{c}$. First, the user power allocations in (3.13) are adjusted using the following fixedpoint equation:

$$
\mathbf{p}=\min \left\{\epsilon_{\mathrm{u}} \mathbf{p}, \mathbf{p}_{\mathcal{S}}^{(\lim )}\right\}
$$

where $\mathbf{p}_{\mathcal{S}}^{(\lim )}$ is the vector of maximum allowed transmission power of BSs and is given by $\mathbf{p}_{\mathcal{S}}^{(\mathrm{lim})}=\mathbf{p}_{\mathcal{S}}^{(\max )} \oslash \mathbf{A}_{\mathcal{S}}$. $\mathbf{A}_{\mathcal{S}}$ contains the number of associated users to each BS where $\mathbf{A}_{\mathcal{S}}=\left(\mathcal{A}_{s}: s \in \mathcal{S}\right)$ and $\oslash$ denotes the Hadamard division. Second, the proposed fixed-point algorithm follows a two-stage procedure to adjust the backhaul 
power allocations in (3.13). Let us consider the maximum allowed backhaul power allocation as:

$$
\zeta=\frac{p_{\mathrm{b}}^{(\max )}-\sum_{i \in \mathcal{A}_{\mathrm{b}}} p_{\mathrm{b}, i}}{D}
$$

where $p_{\mathrm{b}}^{(\max )}$ and $\mathcal{A}_{\mathrm{b}}$ are the transmission power constraint of IAB-donor and the set of associated tUEs with IAB-donor, respectively. Hence, the backhaul power allocations can be adjusted using following fixed-point equation:

$$
\mathbf{p}_{\mathrm{BH}}=\min \left\{\epsilon_{d} \mathbf{p}_{\mathrm{BH}}, \zeta\right\}
$$

if $p_{b, d} \forall d \in \mathcal{D}$ exceeds $\zeta$. Otherwise, the backhaul power allocations are adjusted using the same procedure in (3.14).

The two-stage backhaul power allocation update procedure exploits the transmission power upper bound of the IAB-donor and assures that the inequality constraints of backhaul transmissions in (3.2a) are satisfied, which is critical for UAV-assisted IAB scenarios. It also assures a global convergence to optimum power allocations and user-BS associations after finite number of iterations. Following the same argument in [70, Theorem 3], the proposed fixed-point method converges to a global optimal solution at a geometric rate with $\left\|\mathbf{p}_{c}(i)-\mathbf{p}_{c}^{*}\right\|_{\infty}<C k^{i}$, where $\|\cdot\|_{\infty}$ is the $\ell_{\infty}$-norm, $\mathbf{p}_{c}(i)$ is the combined user and backhaul power allocation vector generated by Algorithm 1 at iteration $i$ with $\mathbf{p}_{c}(i)=\left[\mathbf{p}(i), \mathbf{p}_{\mathrm{BH}}(i)\right], \mathbf{p}_{c}^{*}$ is the optimal power allocations of $\mathcal{P} \mathcal{A}$, and $C>0$ and $0<k<1$ are constants that depend on the problem settings (i.e., channel realizations, user locations and number of users and BSs). The fixed-point algorithm is summarized in Algorithm 1. 


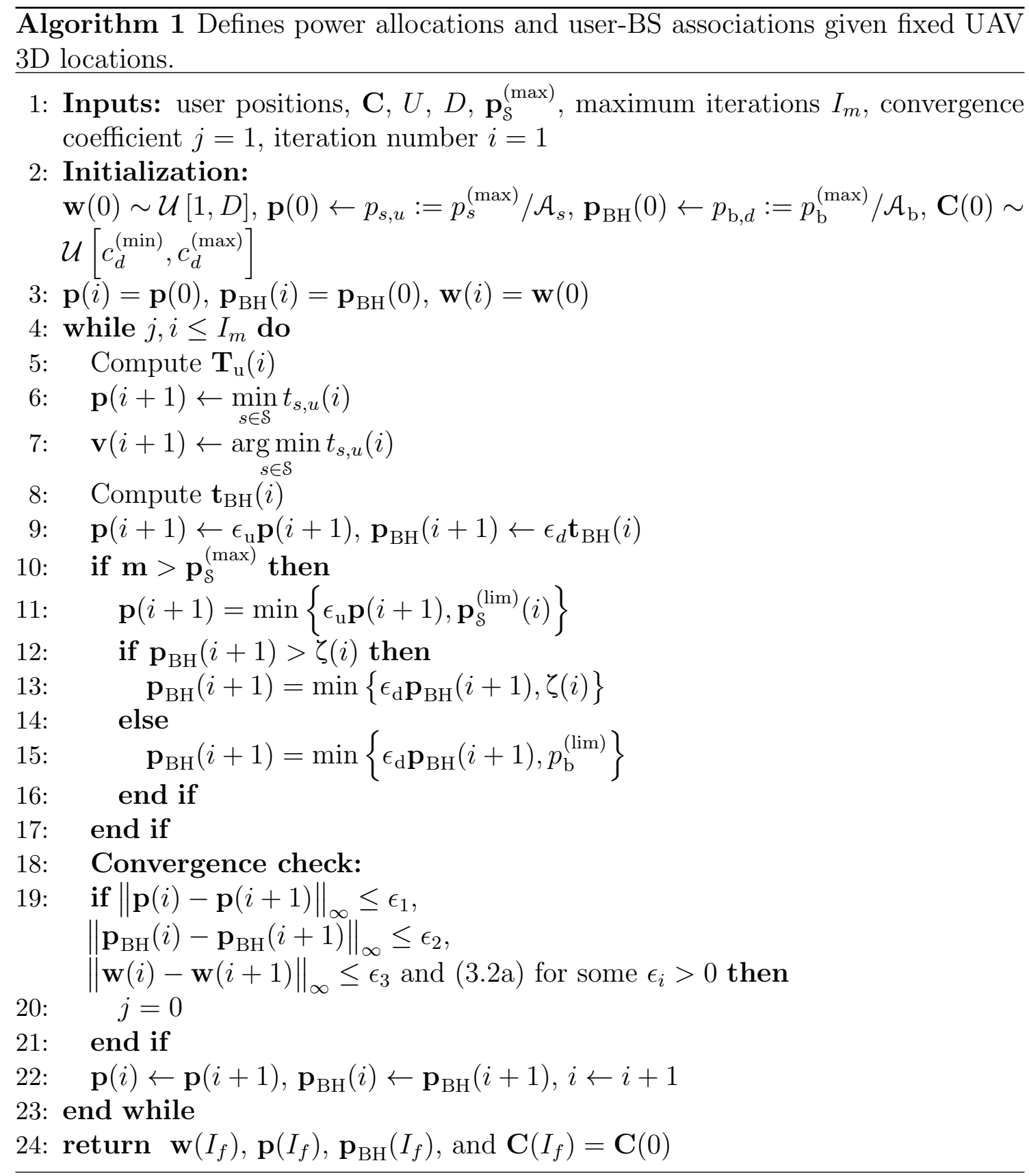




\subsubsection{Particle Swarm Optimization}

We inherit the power allocation and user-BS association results from Algorithm 1 and use them as initial settings for a PSO-based algorithm. Using PSO, we find 3D hovering locations of UAVs and update power allocations accordingly given fixed user-BS associations. PSO is a probabilistic optimization technique that uses the movement characteristics of organisms in a fish school or bird flock to search for set of solutions over a non-convex search space [71,72]. In PSO, each optimization variable is represented by a group of particles, and the swarm is initialized with some values to start searching for a feasible set of solutions. At each iteration, every particle in the swarm moves along the multi-dimensional search space in a probabilistic mechanism taking into account three parameters. First, is the movement velocity of the current iteration. Second, is the distance between the current position and the position of the particle's best objective value, i.e., best local objective value. Third, is the distance between the current position and the position of the swarm's best objective value, i.e., global best objective value, along previous iterations.

Now, let us consider the movement velocities of $M$ particles that represent the $n^{\text {th }}$ variable at $i^{\text {th }}$ iteration as $\mathbf{v}_{n}^{(i)}=\left(v_{n, m}^{(i)}: m \in \mathcal{M}\right)$. Then the matrix of velocities of $M$ particles can be denoted by $\mathbf{V}^{(i)}=\left[\mathbf{v}_{1}^{(i)}, \ldots, \mathbf{v}_{N}^{(i)}\right]^{\top}$, where $\mathbf{V}^{(i)} \in \mathbb{R}^{(N \times M)}$ and $N$ represents the numbers of optimization variables. Similarly, the matrices of current positions and positions of best local objects can be given by $\mathbf{X}^{(i)}=\left[\mathbf{x}_{1}^{(i)}, \ldots, \mathbf{x}_{N}^{(i)}\right]^{\top}$ and $\mathbf{X}_{l}^{(i)}=\left[\mathbf{x}_{1, l}^{(i)}, \ldots, \mathbf{x}_{N, l}^{(i)}\right]^{\top}$, respectively, where $\mathbf{x}_{n}^{(i)}=\left(x_{n, m}^{(i)}: m \in \mathcal{M}\right)$ and $\mathbf{x}_{n, l}^{(i)}=$ $\left(x_{n, m, l}^{(i)}: m \in \mathcal{M}\right)$. Hence, the positions of best local objectives of $M$ particles representing the $n^{\text {th }}$ variable can be given by:

$$
\mathbf{x}_{n, l}^{(i)}=\underset{r \leq i}{\arg \min } \Theta\left(\mathbf{x}_{n}^{(r)}\right)
$$

where the particle's best local objective is defined among previous $r$ iterations. 
Next, let $\mathbf{x}_{g}^{(i)}=\left(x_{n, g}^{(i)}: n \in \mathcal{N}\right)$ represent the positions of global objectives of $N$ variables where $\mathbf{x}_{g}^{(i)} \in \mathbb{R}^{N \times 1}$ and they are given by:

$$
\mathbf{x}_{g}^{(i)}=\underset{m \in \mathcal{M}}{\arg \min } \Theta\left(x_{n, m}^{(i)}\right)
$$

where $\mathbf{x}_{g}^{(i)}$ is the row-minima of $\mathbf{X}^{(i)}$ and $\Theta$ is the weighted fitness function as we will see in 3.21 . Hence, the movement velocity of $(i+1)^{\text {th }}$ iteration can be updated as:

$$
\mathbf{V}^{(i+1)}=\alpha \mathbf{V}^{(i)}+\eta_{1} \mathbf{R}_{1} \odot\left(\mathbf{X}_{l}^{(i)}-\mathbf{X}^{(i)}\right)+\eta_{2} \mathbf{R}_{2} \odot\left(\mathbf{x}_{g}^{(i)}-\mathbf{X}^{(i)}\right)
$$

where the inertia is characterized by $\alpha$ and used to adaptively control the exploration of the optimization process. The cognitive and social learning coefficients are represented by $\eta_{1}$ and $\eta_{2}$, respectively. It is worth noting that, the cognitive and social components in (3.19) control the exploration and exploitation of the optimization process. Specifically, exploitation is set to the highest level when $\eta_{1}=0$ and exploration is set to the highest level when $\eta_{2}=0$. Finally, $\mathbf{R}_{1}, \mathbf{R}_{2} \in \mathbb{R}^{(N \times M)}$ are uniformly distributed numbers between $[0,1]$ and $\odot$ denotes the Hadamard product. Consequently, the position of each particle in $(i+1)^{\text {th }}$ iteration can be updated based on its position in $i^{\text {th }}$ iteration and the movement velocity of $(i+1)^{\text {th }}$ iteration as:

$$
\mathbf{X}^{(i+1)} \leftarrow \mathbf{X}^{(i)}+\mathbf{V}^{(i+1)}
$$

At each iteration we calculate the difference between received and target SINR as $\bar{\gamma}_{u}=\gamma_{u}-\epsilon_{\mathrm{u}}$. Now, let us consider the set of users receiving SINR at access and direct links lower than $\epsilon_{\mathrm{u}}$ as $\vartheta_{\mathrm{u}}=\left\{\bar{\gamma}_{u}: \bar{\gamma}_{u} \in \mathbb{R}^{-1}\right\}$ where $\left|\vartheta_{u}\right|$ denotes the cardinality of $\vartheta_{u}$. Similarly, the set of UAVs receiving SINR at backhaul links lower than $\epsilon_{\mathrm{d}}$ is considered as $\vartheta_{\mathrm{BH}}=\left\{\bar{\gamma}_{\mathrm{BH}}: \bar{\gamma}_{\mathrm{BH}} \in \mathbb{R}^{-1}\right\}$, where $\bar{\gamma}_{\mathrm{BH}}=\boldsymbol{\gamma}_{\mathrm{BH}}-\epsilon_{\mathrm{d}}$. Hence, a weighted fitness function can be composed of the objective function and nonlinear inequality 
constraints in 3.1 and is given by:

$$
\Theta\left(\mathbf{C}, \mathbf{p}, \mathbf{p}_{\mathrm{BH}}\right)=\mathfrak{R}-\left(e_{1}\left|\vartheta_{u}\right|+e_{2}\left|\vartheta_{\mathrm{BH}}\right|\right)
$$

where $e_{1}$ and $e_{2}$ denote penalty parameters and are defined based on the target received QoS at users and UAVs, respectively. $\Theta$ is then evaluated at the current position of each particle and compared with the particle's local best fitness and global fitness of the swarm. The values of $\mathbf{X}_{l}^{(i)}$ and $\mathbf{X}_{g}^{(i)}$ are then updated using (3.17) and (3.18), respectively. Although PSO is easy to implement, compared with other evolutionary computation techniques (see, e.g. [73] and references therein), the computational complexity of swarm optimization increases with the number of optimization variables and constraints. The weighted fitness function in (3.21) reduces the computational complexity of the proposed PSO algorithm and solves the non-linear constrained program in (3.4) independently of the number of optimization constraints in (3.2a).

The time complexity of PSO can be calculated as follows. $T_{\text {comp }}=T_{\text {int }}+\left(T_{\text {eva }}+\right.$ $\left.T_{\text {upd }}\right) \times M$ where, $T_{\text {int }}, T_{\text {eva }}, T_{\text {upd }}, M$ are the computational costs of the initialization, evaluation, velocity and position update of each particle, and the number of particles respectively [74]. Given that the number of optimization variables (i.e., dimensionality of the search space) in Algorithm 2 is $N$, hence, $T_{\text {comp }}=N(1+3 \times M)$. Consequently, we denote the complexity of Algorithm 2 as $\mathcal{O}(N \times M)$. The proposed algorithm converges to a near-optimal solution when the relative change in the best objective function value over the last $I_{c}$ iterations is less than $\epsilon_{4}$. The proposed PSO algorithm and time complexity of the proposed algorithms are summarized in Algorithm 2 and Table 3.1, respectively. 
$\overline{\text { Algorithm } 2 \text { Defines 3D locations of UAVs and updates power allocations accord- }}$ ingly given fixed user-BS associations.

1: Inputs: user positions, $\mathbf{C}(0), U, D, \mathbf{p}_{s}^{(\max )}, N, M, \alpha, \eta_{1}, \eta_{2}, I_{m}, j=1, i=1$

2: Initialization:

$\mathbf{w}(i) \leftarrow \mathbf{w}\left(I_{f}\right), \mathbf{p}(i) \leftarrow \mathbf{p}\left(I_{f}\right), \mathbf{p}_{\mathrm{BH}}(i) \leftarrow \mathbf{p}_{\mathrm{BH}}\left(I_{f}\right)$,

$\mathbf{C}(i) \leftarrow \mathbf{C}\left(I_{f}\right), \mathbf{y}=\left[\operatorname{vec}(\mathbf{C}(i)), \mathbf{p}(i), \mathbf{p}_{\mathrm{BH}}(i)\right]^{\top}$,

$\mathbf{X}^{(i)} \sim \mathcal{U}\left[\epsilon_{1} \mathbf{y}, \epsilon_{2} \mathbf{y}\right], \mathbf{X}_{l}^{(i)}=\underset{r \leq i}{\arg \min } \boldsymbol{\Theta}\left(\mathbf{X}^{(r)}\right)$,

$\mathbf{x}_{g}^{(i)}=\underset{m \in \mathcal{M}}{\arg \min } \Theta\left(x_{n, m}^{(i)}\right)$,

3: while $j, i \leq I_{m}$ do

4: $\quad$ Compute $\mathbf{V}^{(i)}, \mathbf{X}^{(i)}, \mathbf{X}_{l}^{(i)}, \mathbf{x}_{g}^{(i)}, \Theta^{(i)}\left(\mathbf{C}, \mathbf{p}, \mathbf{p}_{\mathrm{BH}}\right)$

5: if $\Theta\left(\mathbf{X}^{(i)}\right)<\Theta\left(\mathbf{X}_{l}^{(i)}\right)$ then

6: $\quad \mathbf{X}_{l}^{(i)} \leftarrow \mathbf{X}^{(i)}$

7: $\quad$ for $n \in \mathcal{N}$ do

8: $\quad$ if $\Theta\left(\mathbf{x}_{n}^{(i)}\right)<\Theta\left(x_{n, g}^{(i)}\right)$ then

9: $\quad x_{n, g}^{(i)} \leftarrow x_{n, m}^{(i)}$

10: $\quad$ end if

11: $\quad$ end for

12: $\quad$ end if

13: Update $\mathbf{V}^{(i+1)}$ and $\mathbf{X}^{(i+1)}$ using $(3.19)$ and $(3.20)$, respectively.

14: $\quad i \leftarrow i+1$

15: Convergence check:

16: $\quad$ if $\frac{\left|\Theta^{(i)}\left(\mathbf{C}, \mathbf{p}, \mathbf{p}_{\mathrm{BH}}\right)-\Theta^{\left(i-I_{c}+1\right)}\left(\mathbf{C}, \mathbf{p}, \mathbf{p}_{\mathrm{BH}}\right)\right|}{\left|\Theta^{(i)}\left(\mathbf{C}, \mathbf{p}, \mathbf{p}_{\mathrm{BH}}\right)\right|} \leq \epsilon_{4}, i>I_{c}$ then

17: $\quad j=0$

18: end if

19: end while

20: return $\mathbf{C}\left(I_{P}\right), \mathbf{p}\left(I_{P}\right), \mathbf{p}_{\mathrm{BH}}\left(I_{P}\right)$, and $\mathbf{w}\left(I_{P}\right)=\mathbf{w}\left(I_{f}\right)$ 
Table 3.1: Time complexity of the proposed algorithms

\begin{tabular}{|c|c|}
\hline Algorithm & Time complexity \\
\hline Fixed-point method & geometric rate with $\left\|\mathbf{p}_{c}(i)-\mathbf{p}_{c}^{*}\right\|_{\infty}<C k^{i}$ \\
\hline PSO & $\mathcal{O}(N \times M)$ \\
\hline
\end{tabular}

\subsubsection{General Solution}

The design parameters of in-band UAV-assisted IAB networks are intertwined together due to the full reuse of wireless channel resources between backhaul and access links, LOS capabilities of UAVs, small inter-site distance and spatial dynamics of user distribution. Hence, we present an iterative algorithm in Algorithm 3 that combines Algorithm 1 and Algorithm 2 to solve problem (3.1). Let us consider $(i>1)$ in Algorithm 3. Hence, the proposed algorithm updates user-BS association vector $\mathbf{w}(i)$ based on the 3D locations matrix $\mathbf{C}(i-1)$. Then, the set of different user-BS associations between current and previous iteration is defined in step 5 . If $\|\mathbf{w}(i)-\mathbf{w}(i-1)\| \geq \epsilon_{1}$ for some $\epsilon_{1} \geq 0$, then, a new set of 3D locations is obtained in step 9. In other words, a new iteration of Algorithm 2 is required for convergence. Similarly, the set of different 3D locations of UAVs and the sum rate difference are obtained in steps 11 and 15 , respectively to define whether new iteration of Algorithm 1 is required for convergence. The proposed algorithm converges to a near-optimal feasible set of solutions after a few iterations.

Our proposed solution for UAV-assisted IAB networks is significantly different compared to the studies in $9,14,15,69,75$. In particular, we consider the mutual dependence between backhaul, direct and access transmissions, inter-cell interference and the mutual dependence between the spatial configurations of UAVs and the spatial dynamics of ground user distribution, which are significantly challenging in UAV-based cellular scenarios. 


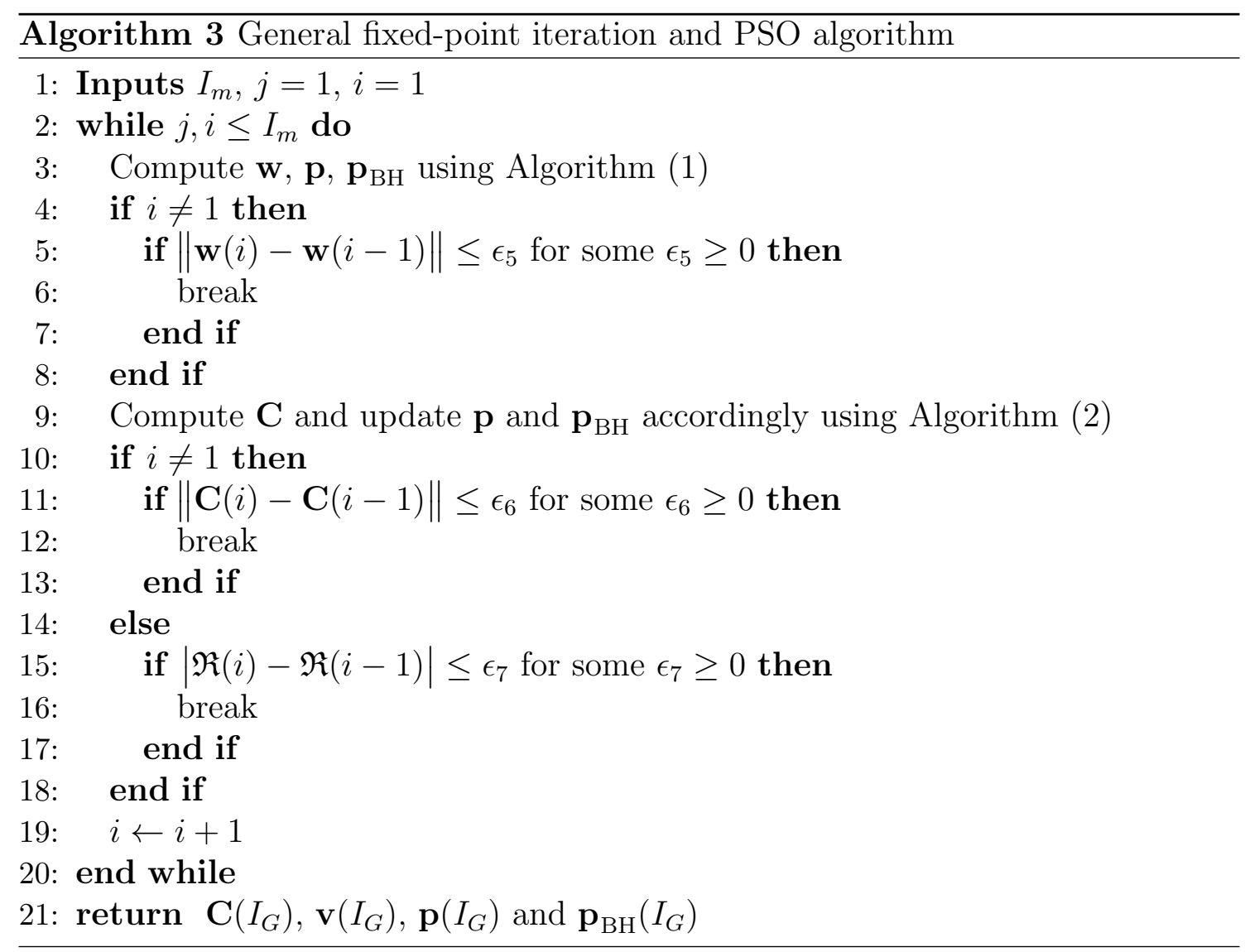




\section{CHAPTER 4 \\ NUMERICAL RESULTS}

In this chapter, we numerically evaluate the performance gains of using UAVs as IAB-nodes in in-band IAB networks. Specifically, we use Algorithm (3) and Monte Carlo simulations to study the achievable gains in received downlink SINR and overall network sum rate. In doing so, we define two use cases for the spatial configurations of UAVs based on the spatial distribution of ground users and compare their performance with the baseline scenario, in which, UAVs are not used. In the baseline scenario, we define the downlink access power allocations as $p_{\mathrm{b}, u}^{*}=\left(\frac{1}{\lambda}-\frac{N_{0}}{\left|h_{\mathrm{b}, u}\right|^{2}}\right)^{+}$, where $p_{\mathrm{b}, u}^{*}$ is the waterfilling power allocation and $\lambda$ satisfies $\frac{1}{U} \sum_{u \in U}\left(\frac{1}{\lambda}-\frac{N_{0}}{\left|h_{\mathrm{b}, u}\right|^{2}}\right)^{+}=p_{\mathrm{b}}^{(\max )}$. Each UAV is equipped with a single transmit antenna due to the limited volume, weight, and payload of drone IAB-nodes. The channel realizations in (2.1) and (2.2), and the spatial distribution of ground users are randomly updated every Monte Carlo simulation. The simulation parameters of both scenarios are summarized in Table 4.1 .

\subsection{Dual Clusters Spatial Distribution of Cellular Users}

In this scenario, we study the use case where users are concentrated in the center of a single hotspot, e.g., music festivals and sports events as depicted in Fig. 4.1. In such scenarios, it is better for aUEs to be associated with a single DAA rather than being associated with distributed UAVs (see Chapter 4). Although IAB-donor allows for multi-user MIMO transmissions at backhaul links, it adopts SISO downlink transmissions to tUEs. Hence, we can fairly evaluate the performance of using DAA with different spatial distributions of ground users (see Section 4.2) . Fig. 4.2 shows that the average received SINR of ground users is enhanced by more than 30 
Table 4.1: Simulation parameters

\begin{tabular}{|c|c|c|}
\hline Settings & Distributed UAVs & Single DAA \\
\hline IAB-donor TM: direct links & SISO & SISO \\
\hline IAB-donor: backhaul links & MIMO & MIMO \\
\hline IAB-donor: TX antennas & 64 & 64 \\
\hline Number of UAVs & 4 & 4 \\
\hline UAV: TX antennas & 1 & 1 \\
\hline DAA TM: access links & - & MIMO (4 layers) \\
\hline UAV TM: access link & SISO & - \\
\hline Number of users & 25 & 25 \\
\hline$f_{c}$, BW, $p_{\mathrm{b}}^{(\max )}, p_{d}^{(\max )}$ & $2 \mathrm{GHz}, 20 \mathrm{MHz}, 46 \mathrm{dBm}, 36 \mathrm{dBm}$ \\
\hline$\sigma^{2}, \epsilon_{\mathrm{u}}, \epsilon_{\mathrm{d}}$ & $-104 \mathrm{dBm}, 3 \mathrm{~dB}, 3 \mathrm{~dB}$ \\
\hline$M, \alpha, \eta_{1}, \eta_{2}$ & \multicolumn{2}{|c|}{$200,[0.1,1.1], 1.49,1.9$} \\
\hline
\end{tabular}

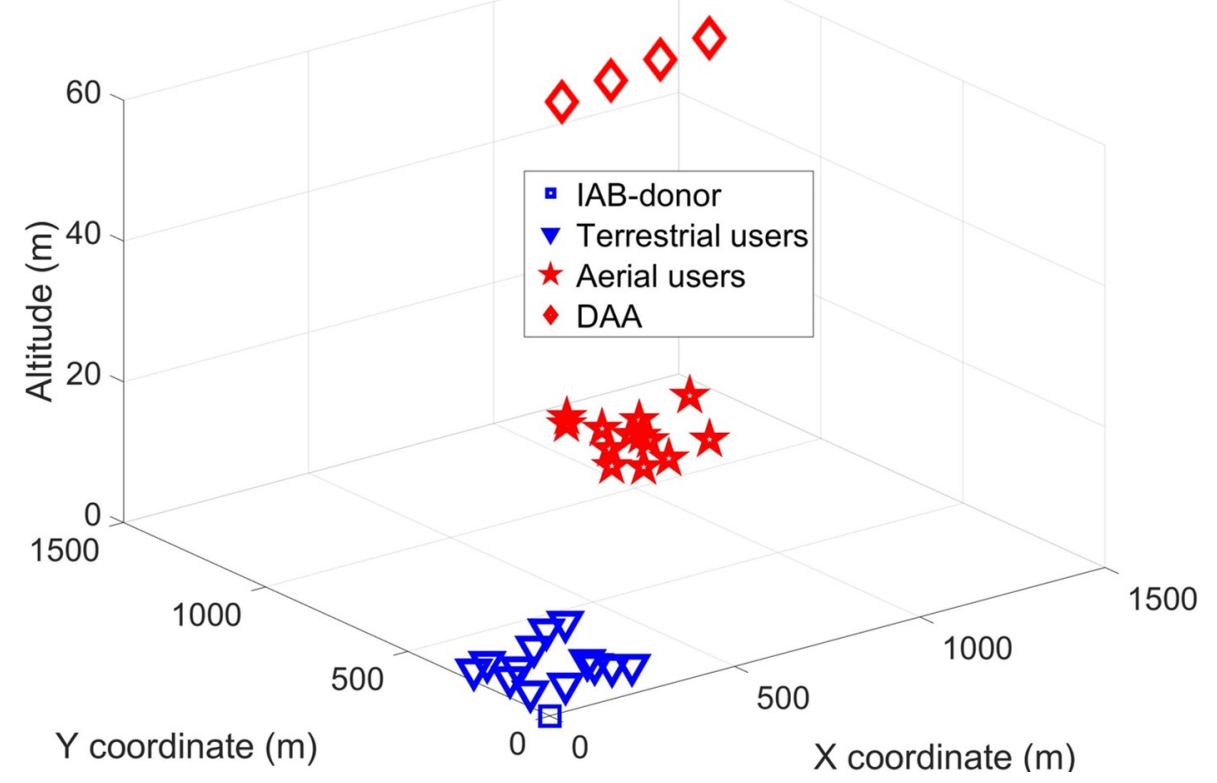

Figure 4.1: Dual clusters: spatial configurations of DAA. 


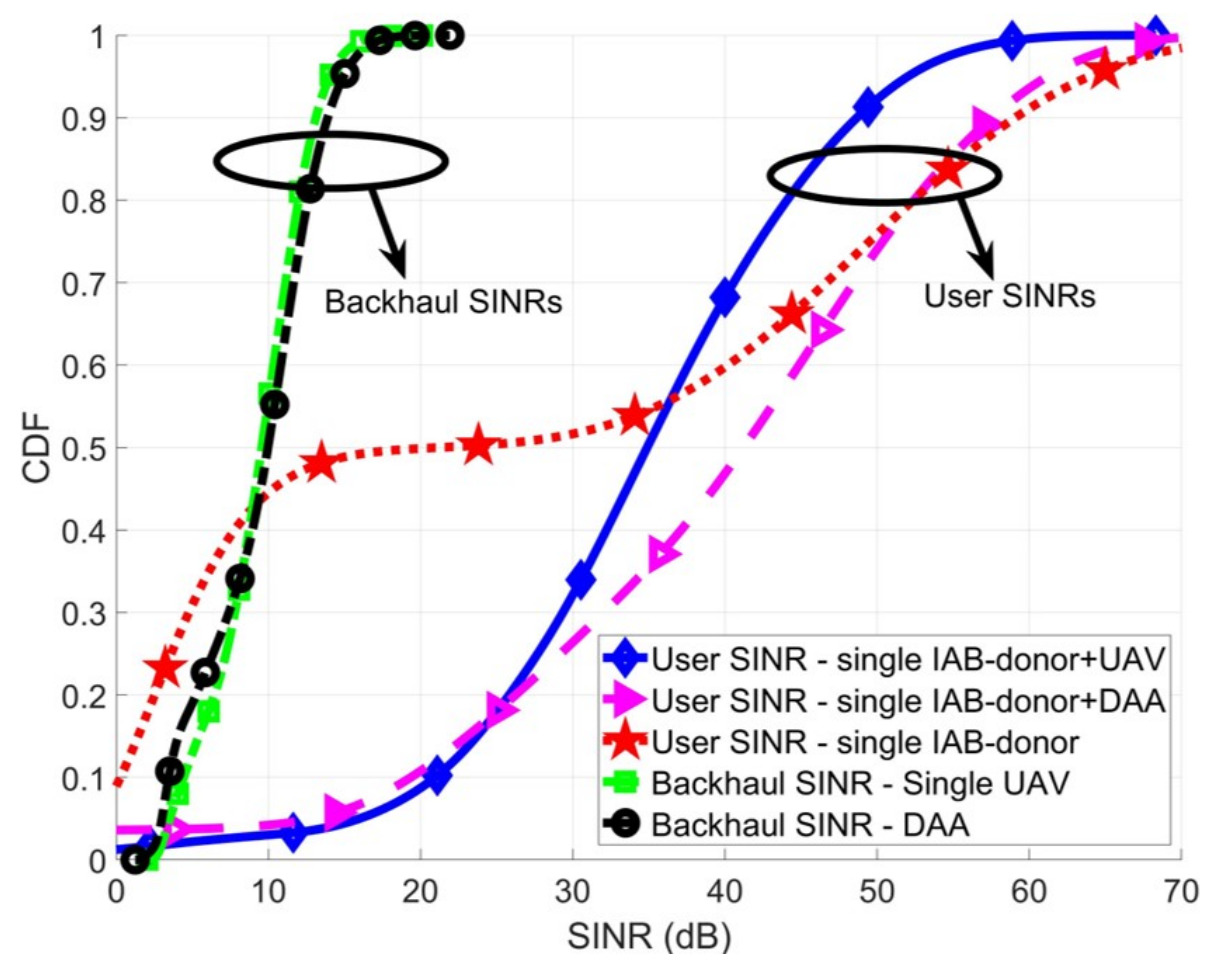

Figure 4.2: Dual clusters: received downlink SINR.

dB after using DAA. Further, it reveals that the received SINR of tUEs is slightly decreased in order to increase the SINR of aUEs. Fig. 4.2 also shows how the spatial configuration of UAVs is intertwined with the spatial distribution of ground users. In that, the received SINR is significantly improved when UAVs are configured as DAA compared with the spatial configuration of distributed UAVs. Finally, Fig. 4.2 shows that the received SINR at backhaul links is consistent with the inequality constraints 3.2a) and 3.6a).

The enhancement in the received downlink throughput in Fig. 4.3 is consistent with the results in Fig. 4.2. It is worth noting that the received throughput at aUEs is higher than that of tUEs after using the DAA. This is because the use of DAA allows for $D$-fold spatial multiplexing gain. Generally, the DAA exploits full spectrum resources to transmit $D$ independent spatial streams to $D$ users per SDMA 


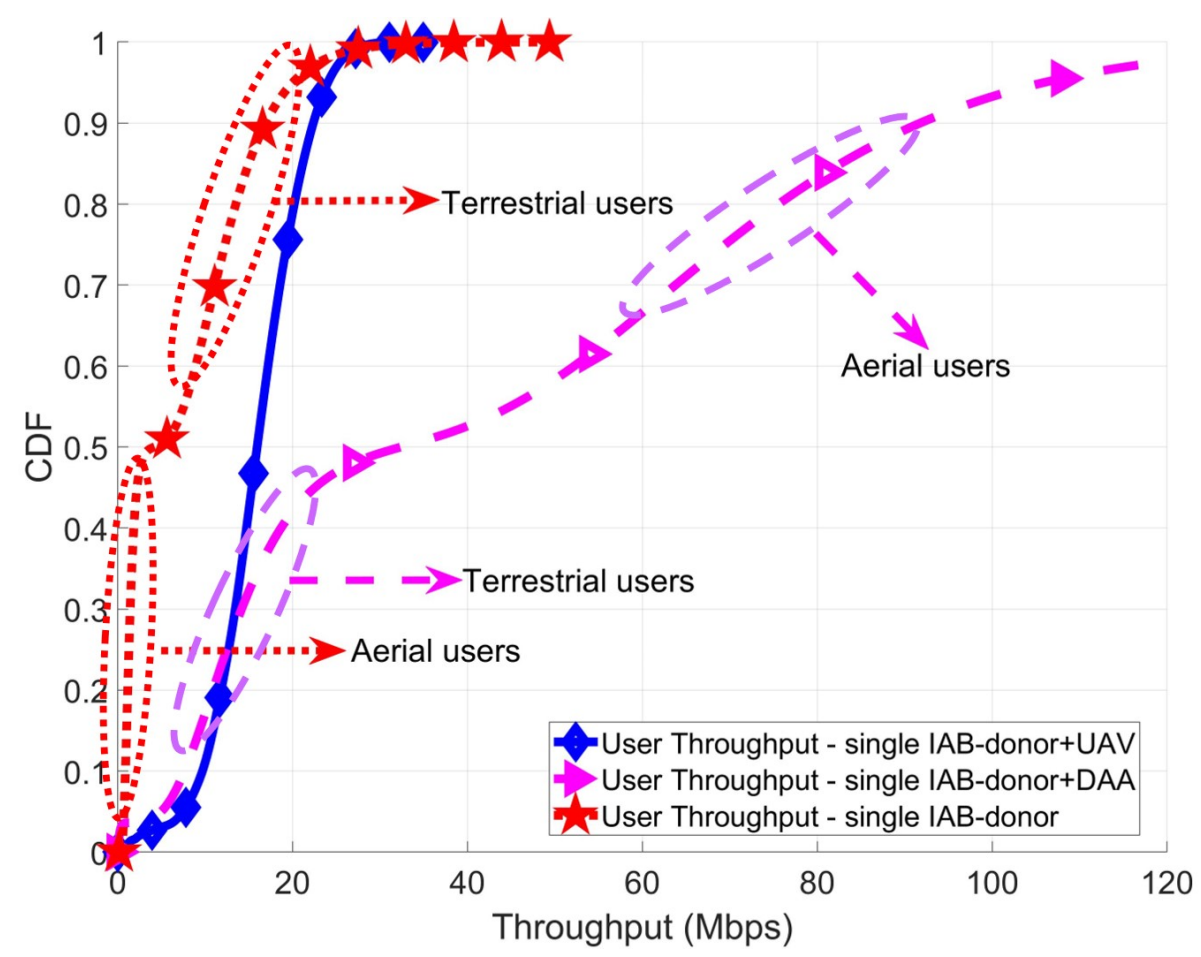

Figure 4.3: Dual clusters: received downlink user throughput.

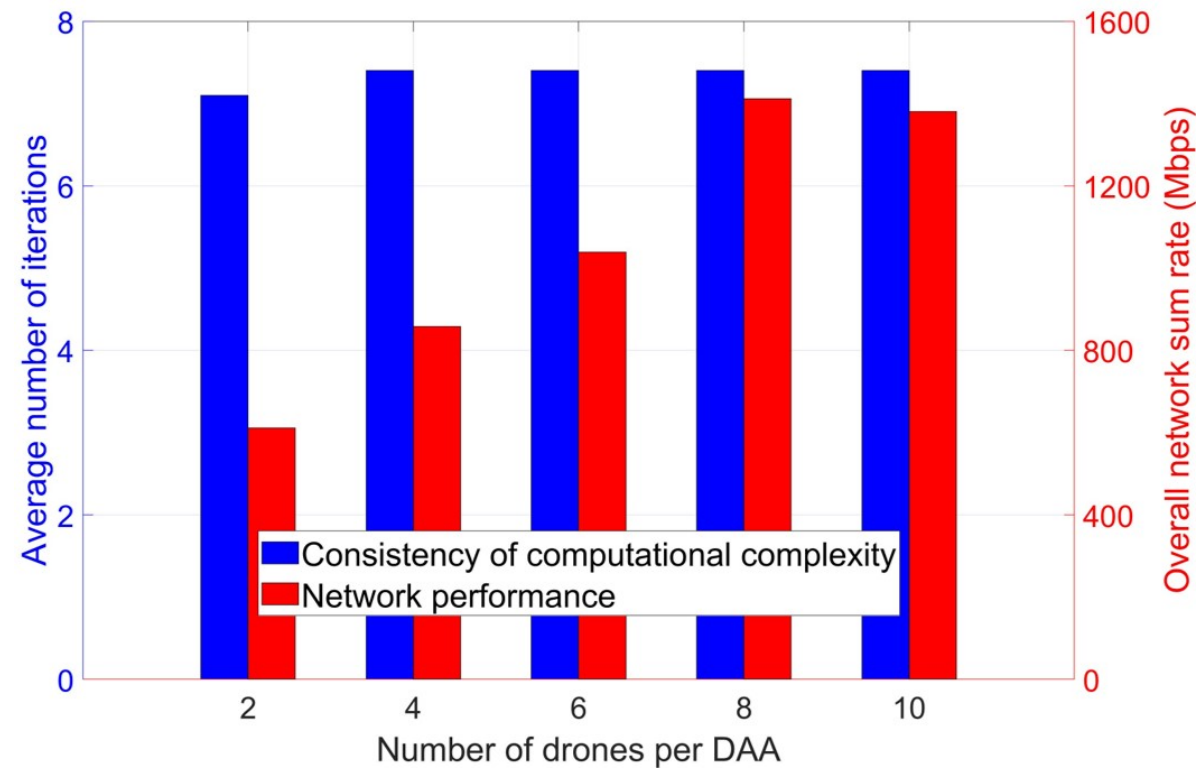

Figure 4.4: The computational complexity of Algorithm 2 with respect to the number of drones per DAA. 
group. Hence, the allocated spectrum resources to aUEs are now much higher than those allocated to tUEs. Consequently, Fig. 4.3 reveals that UAVs can be used as DAA in in-band IAB scenarios not only for coverage enhancement but also for capacity boosting. Fig. 4.3 also shows that offloading aUEs from IAB-donor to DAA helps to improve the downlink throughput of tUEs. Finally, it is worth noting that the number of UAVs per DAA can be increased based on the capacity demands, while ensuring the same computational complexity of 3.5 .

Fig. 4.4 demonstrates the consistency of the computational complexity of the proposed algorithm for a larger number of UAVs. The number of iterations is

slightly increased due to the increased dimensions of $\mathbf{p}_{\mathrm{BH}}$ in 3.8 . It also shows how the overall network performance is directly proportional to the number of UAVs when they are spatially configured as DAA. Further, it reveals that the network performance decreases at high number of UAVs due to the increased levels of mutual interference between backhaul and access links.

\subsection{Multiple Clusters Spatial Distribution of Cellular Users}

In this scenario, users are normally distributed into multiple clusters in the designated coverage area as depicted in Fig. 4.5. Fig. 4.6 shows that the received SINR is enhanced after deploying the DAA in an optimized 3D location between the user clusters. Further, it is significantly enhanced by more than $20 \mathrm{~dB}$ when UAVs are used as distributed hovering IAB-nodes. These results are consistent with the results in Fig. 4.2, in which, the received SINR at tUEs is slightly decreased in order to increase the received SINR at aUEs. In addition, Fig. 4.6 shows that the received SINR at backhaul links is consistent with the inequality constraints 3.2a and (3.6a). 


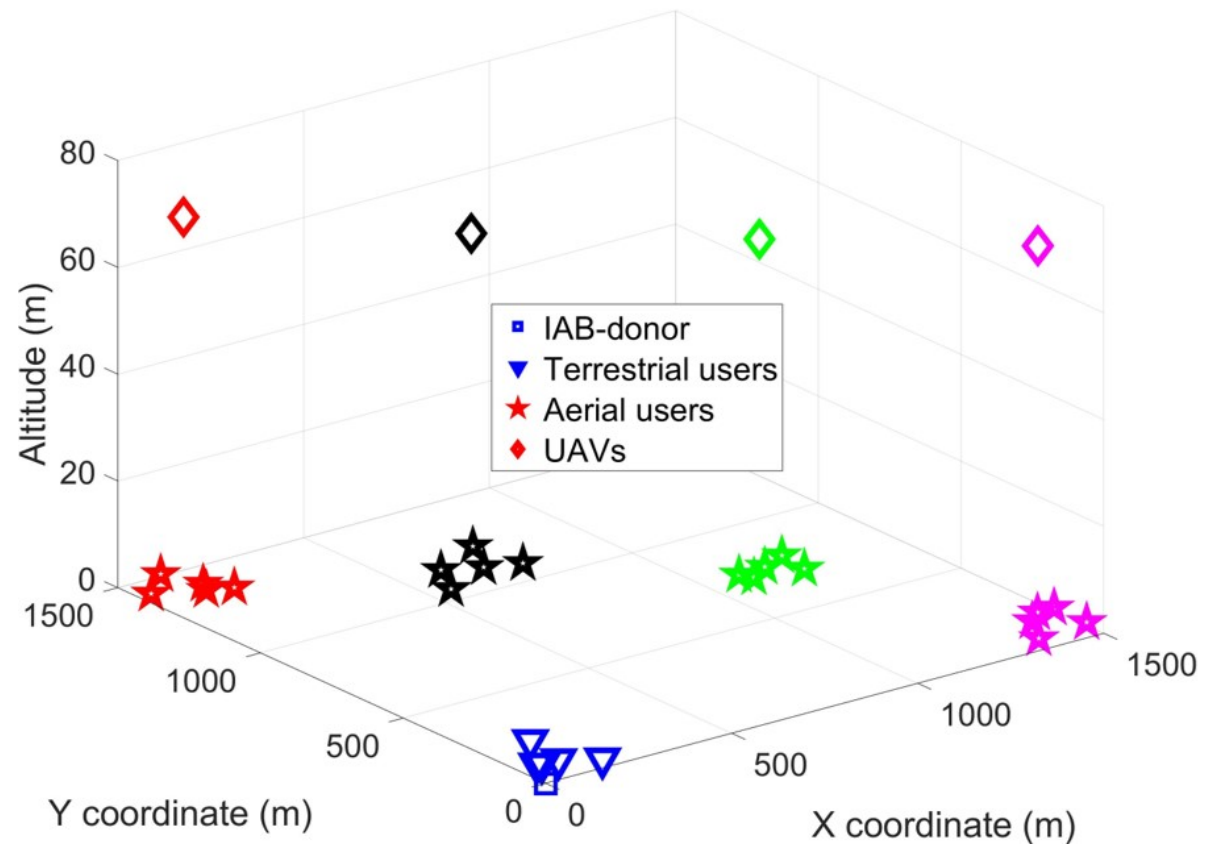

Figure 4.5: Multiple clusters: spatial configurations of UAVs.

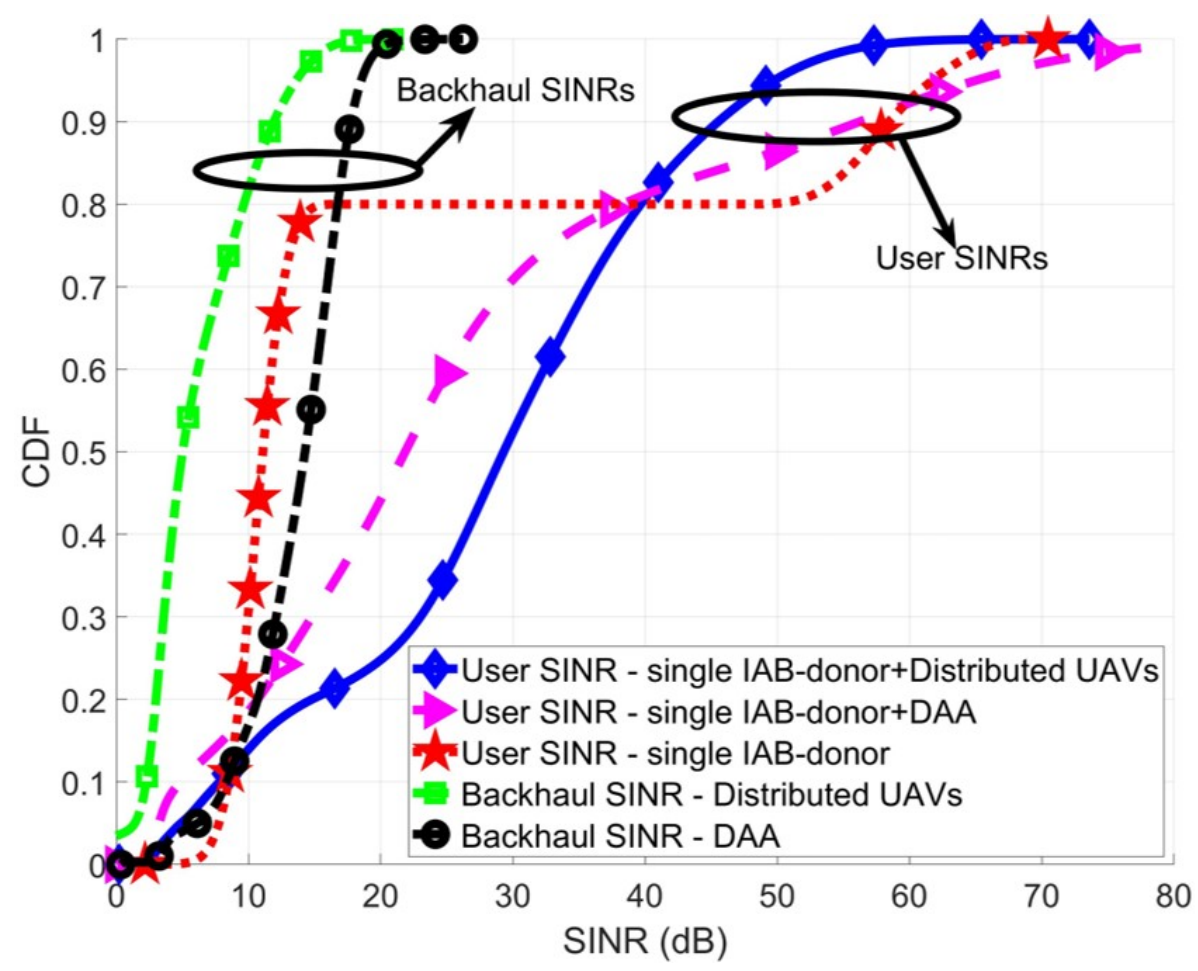

Figure 4.6: Multiple clusters: received downlink SINR. 


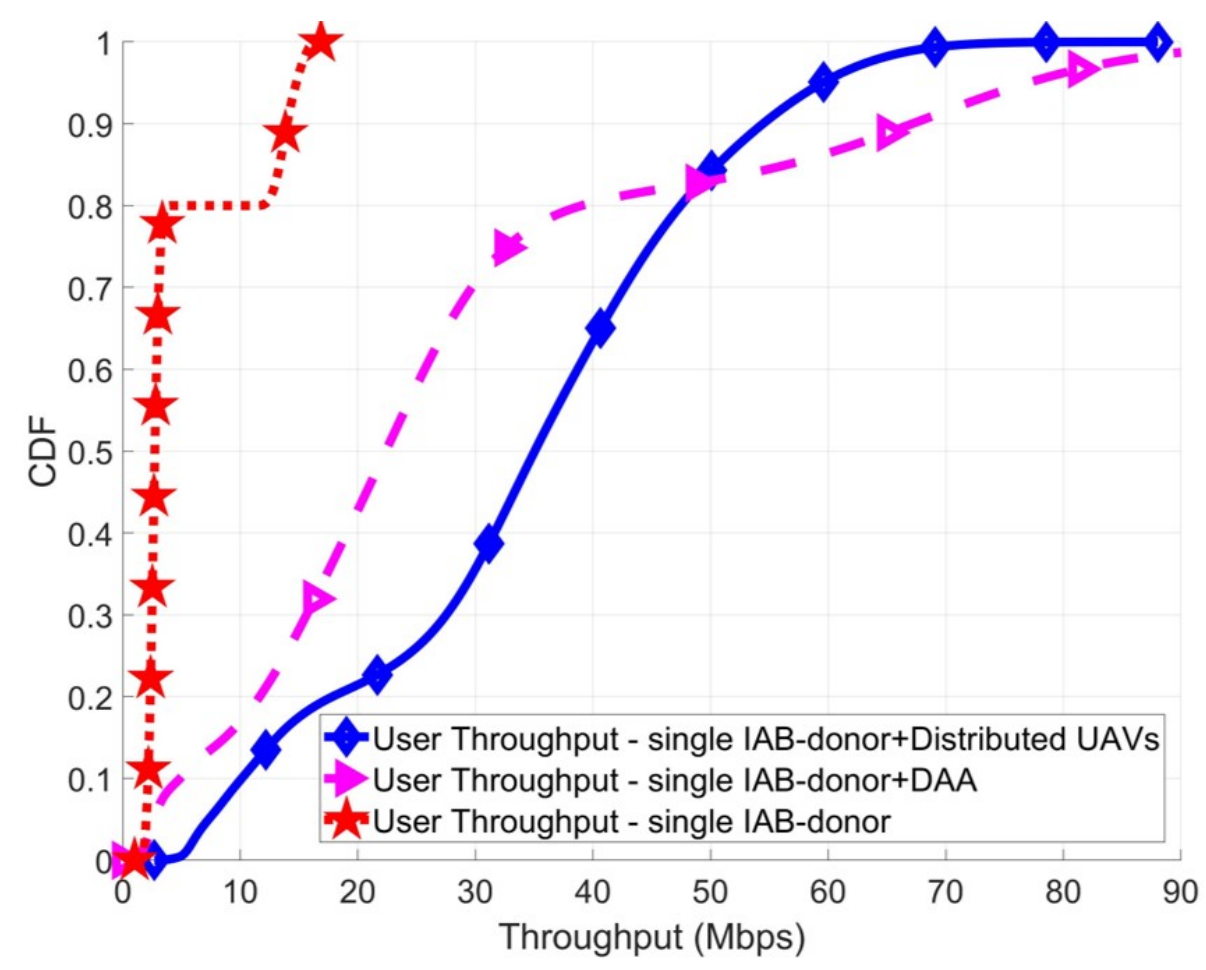

Figure 4.7: Multiple clusters: received downlink user throughput.

Fig. 4.7 shows that the enhancement in the received downlink throughput is consistent with the results in Fig. 4.6. It is worth noting that downlink throughput performance of distributed UAVs outperforms that of DAA, although using DAA allows for $D$-fold spatial multiplexing gain. This is because, the low received downlink SINR at aUEs, i.e., users associated with DAA. In particular, the intermediate 3D deployment of DAA between the distributed clusters results in suboptimal directivity towards aUEs. In contrast, the DAA gains are maximized when it is fully directed to serve aUEs concentrated in a single hotspot (as discussed in Section 4.1). Fig. 4.8 presents the favorable spatial configuration of UAVs based on the spatial distribution of ground users. 


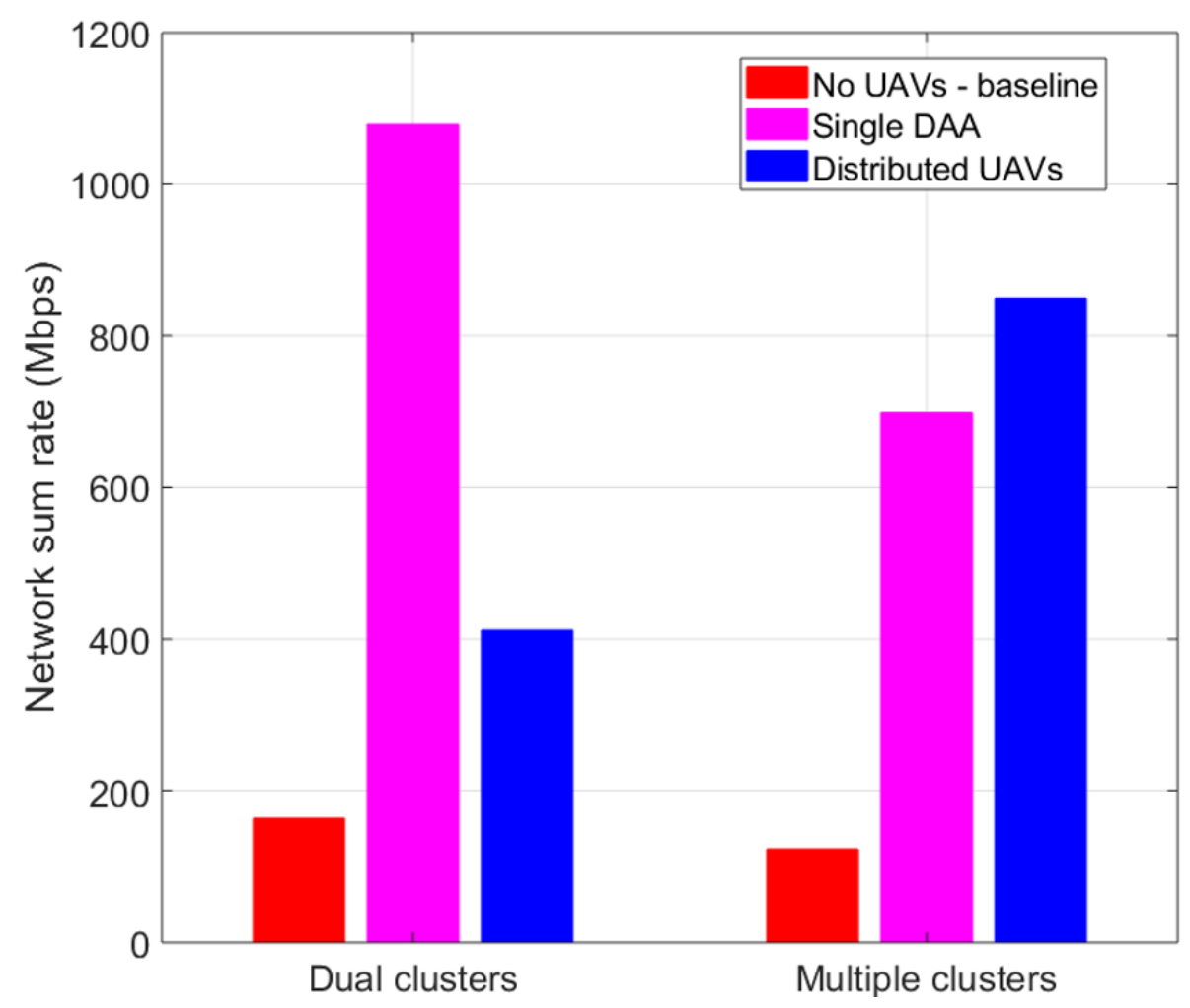

Figure 4.8: Favorable spatial configurations of UAVs.

\subsection{Convergence Analysis of the PSO Algorithm}

As mentioned in Section 3.3.2, the proposed PSO solution in Algorithm 2 converges to a near-optimal solution when the relative change in the best objective function value over the last $I_{c}$ iterations is less than $\epsilon_{4}$. In this section, we analyze the convergence results of the proposed PSO algorithm at different spatial configurations of UAVs. Fig. 4.9 shows that the fitness function $\Theta\left(\mathbf{C}, \mathbf{p}, \mathbf{p}_{\mathrm{BH}}\right)$ of the proposed PSO algorithm converges to a near-optimal solution after a few number of iterations when UAVs are spatially configured as DAA. It also shows that the time complexity of the proposed PSO algorithm can be significantly improved by increasing the value of $\epsilon_{4}$ without decreasing the accuracy of the optimized set of solutions.

On the other hand, Fig. 4.10 shows that decreasing $\epsilon_{4}$ will impact the accuracy of the optimized set of solutions when UAVs are spatially configured as distributed 


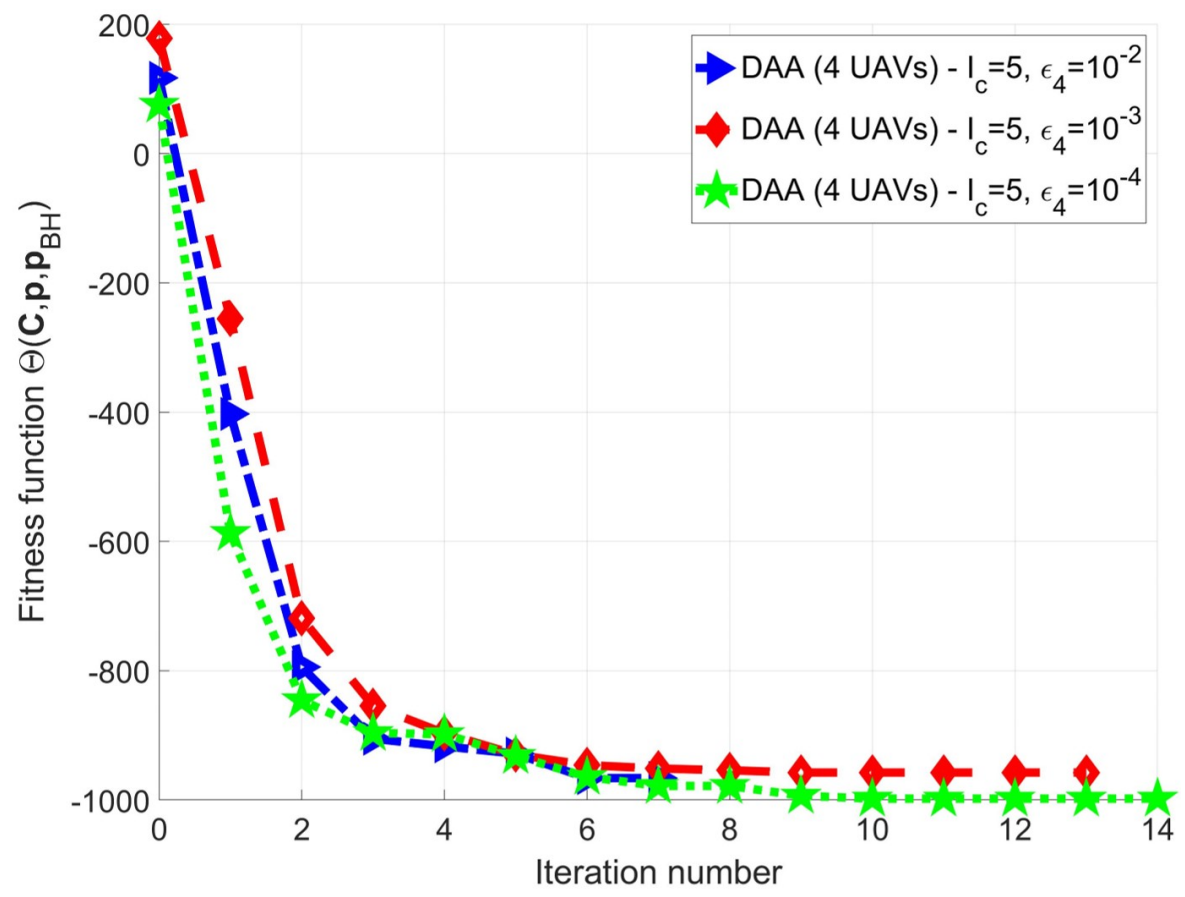

Figure 4.9: Dual clusters: PSO convergence.

UAVs (i.e., at a larger number of optimization variables). It is worth noting that the convergence window size (i.e., $I_{c}$ ) is required to be increased as the number of the optimization variables increases to assure the convergence to a near optimal solution. Hence, we use $I_{c}=5$ and $I_{c}=20$ when UAVs are spatially configured as DAA (Fig. 4.9) and as distributed UAVs (Fig. 4.10), respectively. Finally, Figs. 4.9 and 4.10 demonstrate that Algorithm 2 converges to a near-optimal solution in a fewer number of iterations when UAVs are spatially configured as DAA. In other words, the proposed PSO algorithm converges faster to a near-optimal solution when the number of the optimization variables is smaller. 


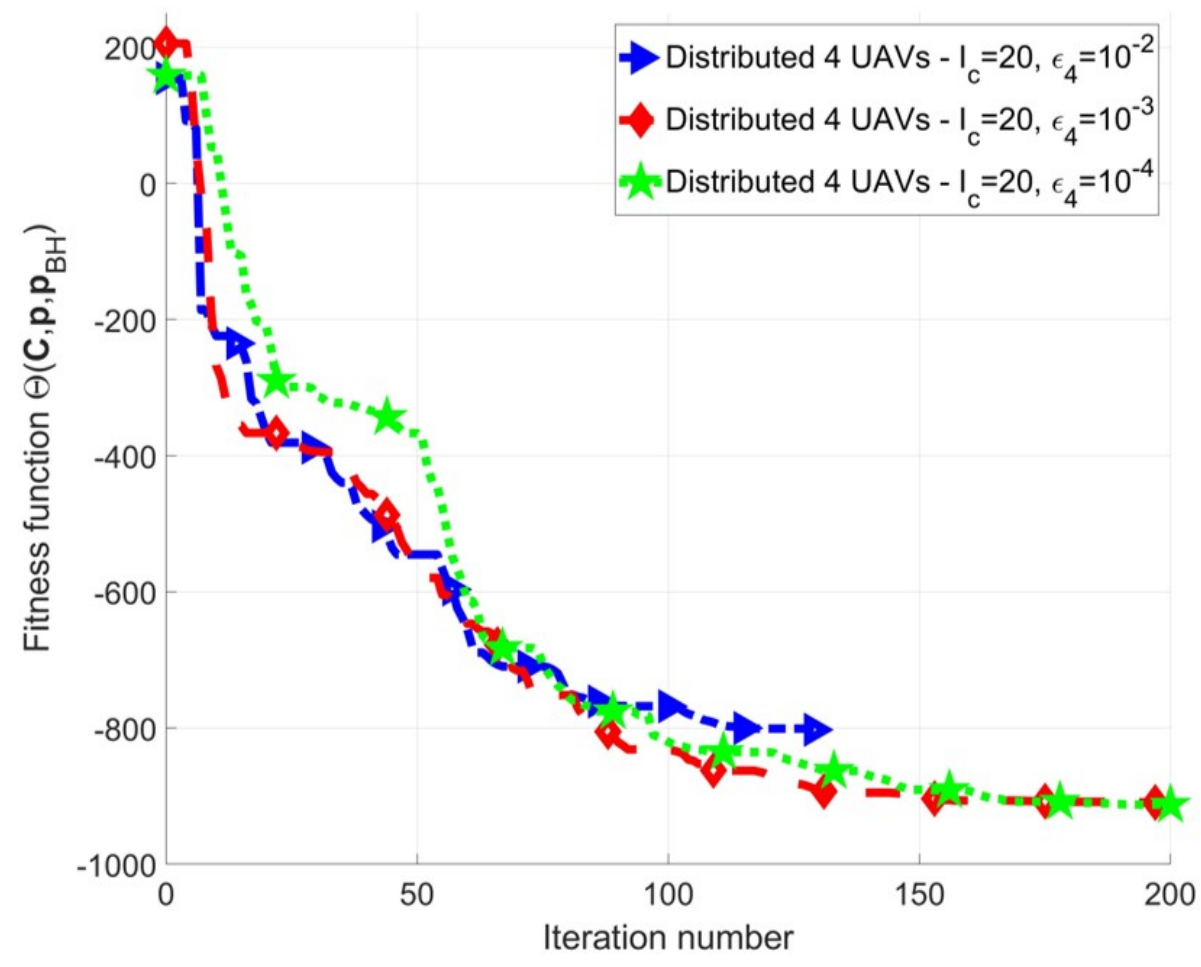

Figure 4.10: Multiple clusters: PSO convergence.

\subsection{Numerical Evaluation of Reversed Algorithm 3}

In Section 3.3.3, we presented an iterative solution in Algorithm 3 that combines Algorithms 1 and 2 to solve the master optimization problem (9). In this section, we present the numerical results of the reversed version of Algorithm 3 (i.e., to optimize the 3D locations of UAVs at first and the user-BS associations at second). We carried out the optimization steps in a reversed order to find the optimized set of solutions when the cellular users are spatially distributed into multiple clusters (see Fig. 7). Our numerical results in Figs. 4.11 and 4.12 show that the reversed and regular optimization orders converge to almost the same results. Essentially, the optimized solution of (9) does not depend on the order of the optimization steps, given that the proposed Algorithm 3 converges to a near-optimal set of solutions after a few iterations. However, it is worth noting that the time complexity of the 


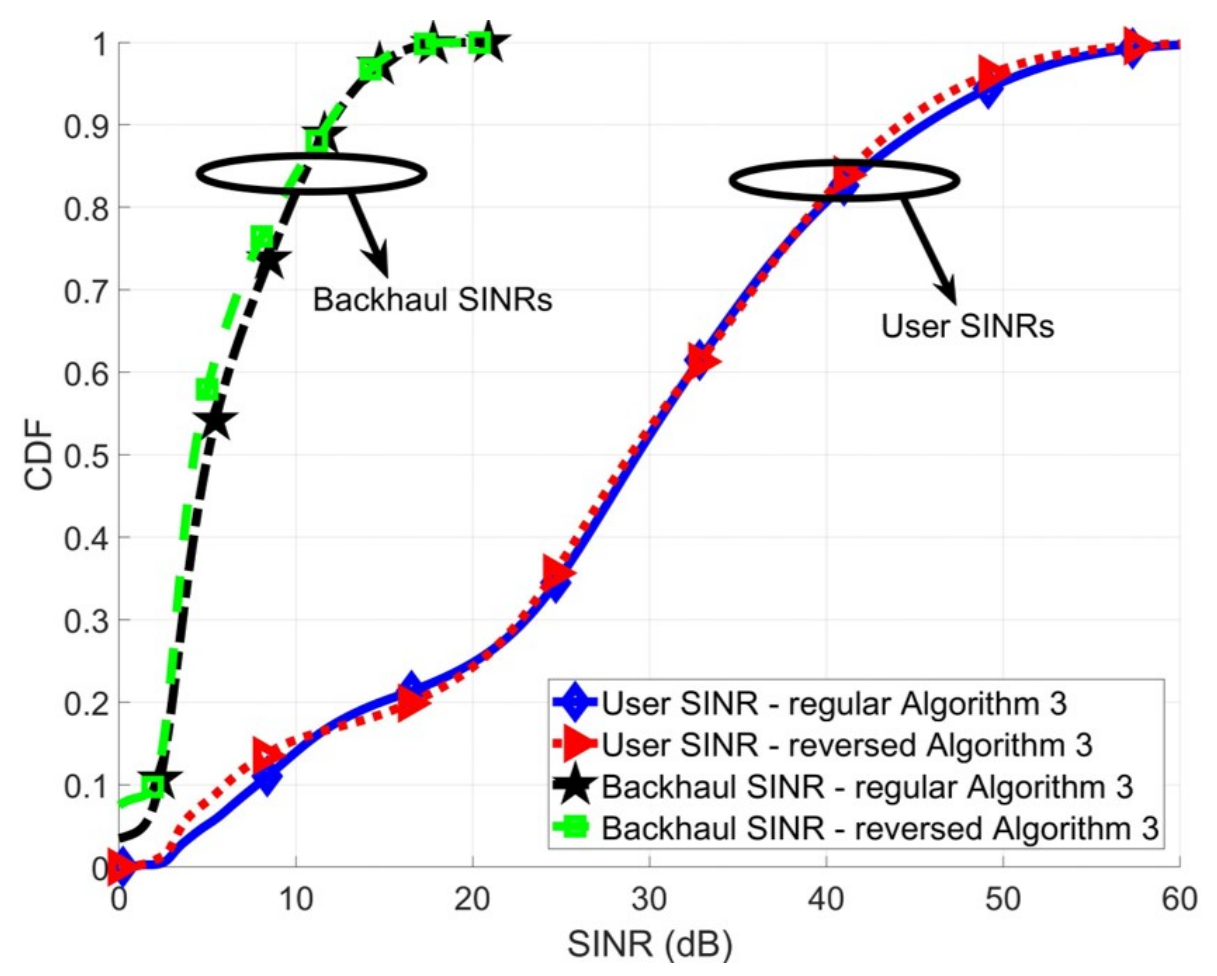

Figure 4.11: Reversed Algorithm 3: downlink SINR.

reversed optimization order is always higher than that of the regular order. This is because the PSO algorithm (Algorithm 2) is more time-consuming than the fixedpoint method (Algorithm 1). Generally, the number of required PSO iterations in the reversed optimization order is higher than that of the regular order.

\subsection{Generic Spatial Distribution of Cellular Users}

In this section, we numerically evaluate the performance of generic spatial distribution of cellular users. Specifically, a fraction of users are uniformly distributed within the coverage area (i.e., non-clustered users) and others are distributed into multiple hotspots (i.e., clustered users) as depicted in Fig. 4.13. Fig. 4.14 shows that the overall received downlink SINR is slightly decreased when cellular users 


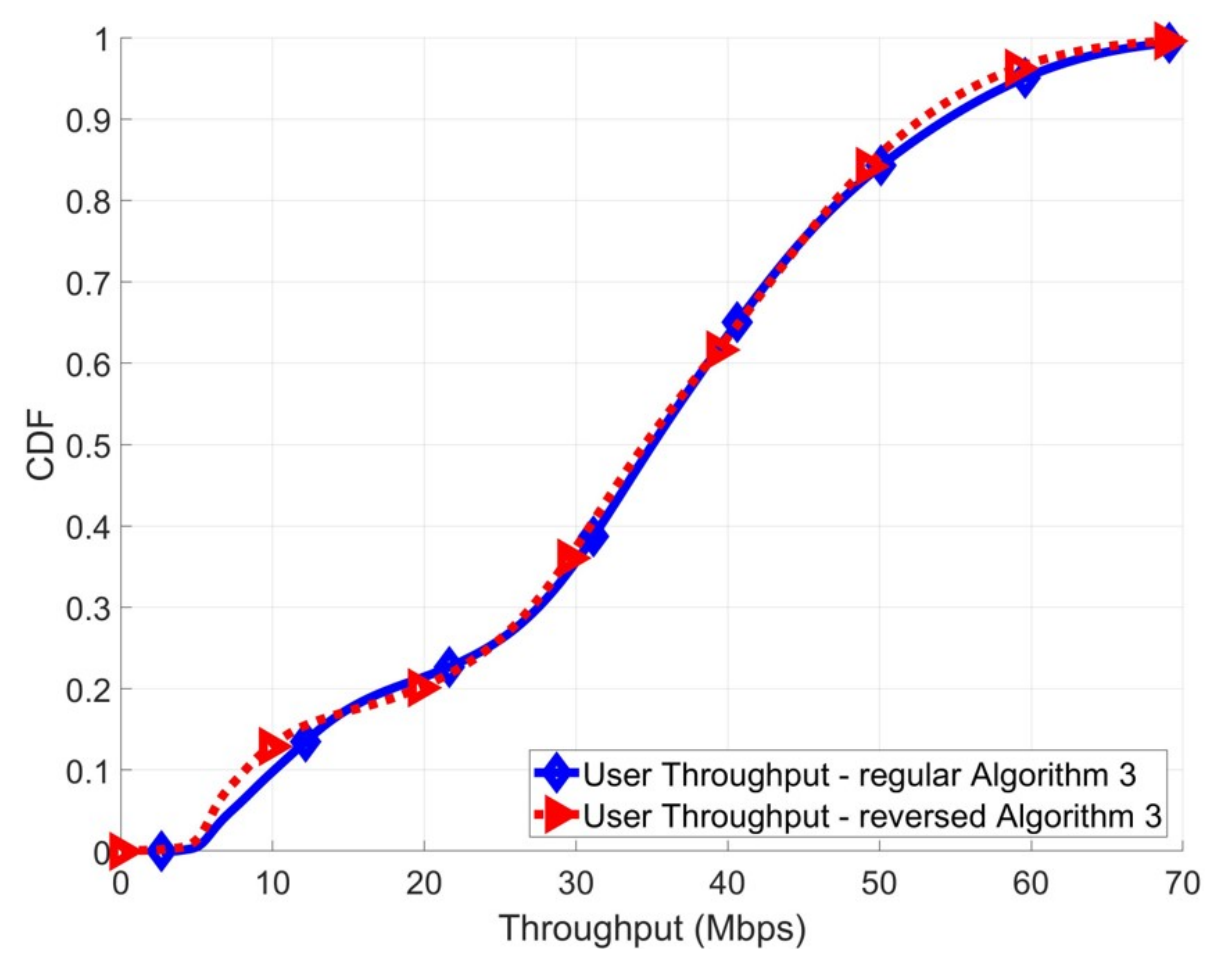

Figure 4.12: Reversed Algorithm 3: downlink throughput.

are spatially distributed as clustered and non-clustered users compared with the clustered distribution scenario. Essentially, the received downlink interference levels at non-clustered users are higher than those received at clustered users due to their intermediate locations between the hotspots. Thus, the overall SINR performance is decreased by $\approx 2 \mathrm{~dB}$ as shown in Fig, 4.14. It is worth noting that backhaul performance is almost the same in both scenarios. This is because the spatial distributions of UAVs are almost the same (i.e., the 3D deployment of UAVs). Fig. 4.15 shows that the downlink throughput is also decreased when the cellular users are spatially distributed into clustered and non-clustered users, which is consistent with the SINR degradation in Fig. 4.14. Our numerical results in this section reveal that the performance of the proposed algorithms is directly proportional to the heterogeneity of the spatial distribution of cellular users (i.e., performance gain increases with more clustered users). 


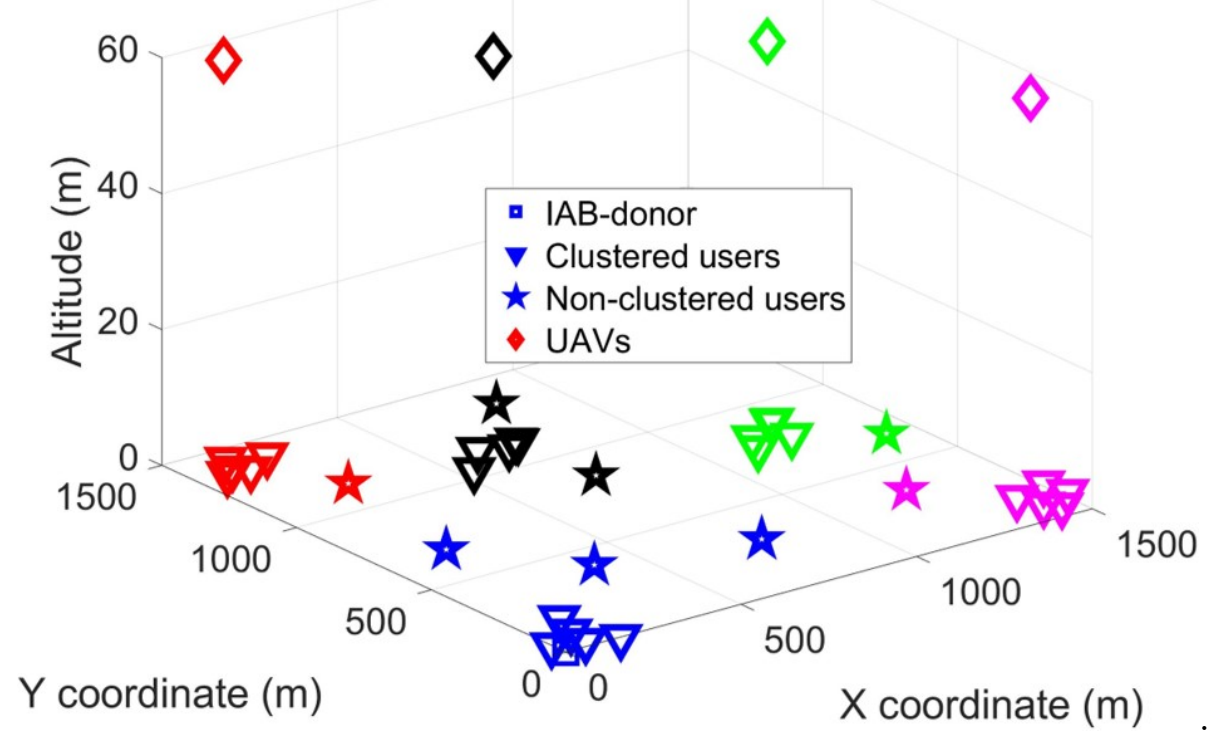

Figure 4.13: Generic spatial distribution of cellular users.

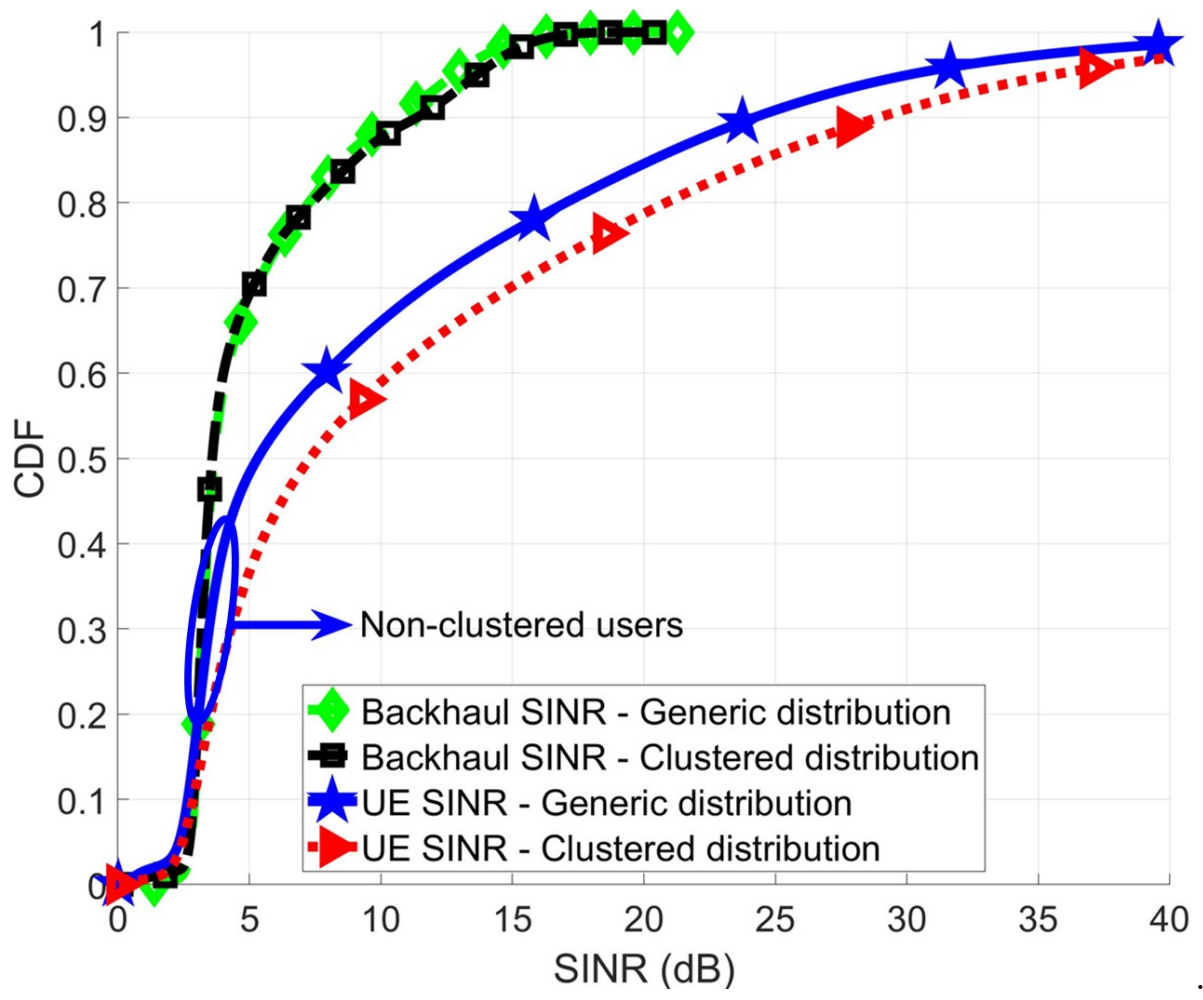

Figure 4.14: Generic distribution: downlink SINR. 


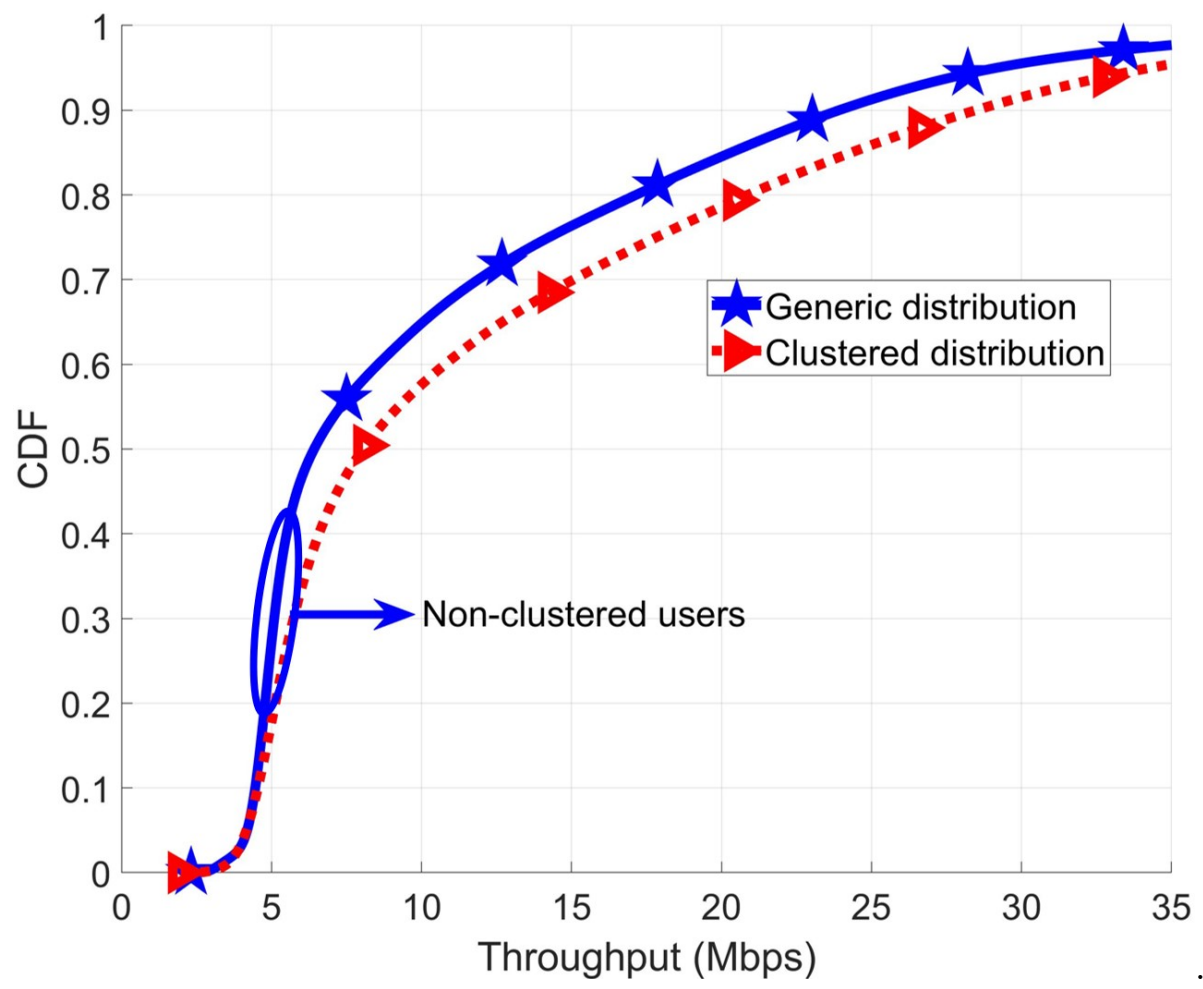

Figure 4.15: Coupled distribution: downlink backhaul SINR. 


\section{CHAPTER 5 \\ CONCLUDING REMARKS}

In this thesis, we propose an UAV-based interference management algorithm to optimize the performance of in-band UAV-assisted IAB networks. In-band IAB network architecture allows for tighter interworking between access and backhaul links, making it a promising solution to meet the requirements of fast and easily scalable deployment of next-generation cellular networks. The problem is cast as network sum rate maximization problem. In which, we exploit fixed-point method and PSO to jointly optimize user-BS associations, downlink power allocations and the 3D spatial configurations of UAVs, taking into account the full reuse of wireless channel resources between backhaul, direct and access links, inter-cell interference and LOS capabilities of UAVs. Further, we investigate the mutual dependence between the spatial configurations of UAVs in the sky and the spatial dynamics of

ground user distribution. In particular, we consider distributed UAVs and DAA as different spatial configurations of UAVs.

Our numerical results show that the spatial configuration of distributed UAVs outperforms that of the DAA by $21.6 \%$ in terms of the overall network sum rate when the ground cellular users are normally distributed into multiple bad-coverage areas. On the other hand, the spatial configuration of the DAA outperforms that of distributed UAVs by $161.9 \%$ when the ground cellular users are concentrated in a single bad-coverage area. Moreover, we show that the proposed algorithm is of low complexity and independent of the number of UAVs when they are spatially configured as DAA. We also analyze the convergence results of the proposed PSO algorithm and show how PSO settings can be adjusted to converge to the same nearoptimal set of solutions in fewer number of iterations. We discuss the robustness of the proposed iterative algorithm against the order of the optimization steps and show 
that it converges to same optimized set of solutions irrespective of the order of the optimization steps. Furthermore, our numerical results reveal that the performance of the proposed algorithms is directly proportional to the heterogeneity of the spatial distribution of cellular users (i.e.,performance gain increases with more clustered users). 


\section{BIBLIOGRAPHY}

[1] T. K. Vu, M. Bennis, M. Debbah, and M. Latva-aho, "Joint path selection and rate allocation framework for $5 \mathrm{G}$ self-backhauled mmWave networks," IEEE Trans. Wireless Commun., pp. 1-1, Mar. 2019.

[2] Z. Gao, L. Dai, D. Mi, Z. Wang, M. A. Imran, and M. Z. Shakir, "MmWave massive-MIMO-based wireless backhaul for the 5G ultra-dense network," IEEE Wireless Commun., vol. 22, no. 5, pp. 13-21, Oct. 2015.

[3] Technical Specification Group Radio Access Network, "Study on integrated access and backhaul," 3GPP, Tech. Rep. 3GPP TR38.874 v16.0.0, Dec. 2018.

[4] AT\&T, Qualcomm, Samsung, "Study on integrated access and backhaul for NR," 3GPP, Tdoc 3GPP RP-171880, Sep. 2017.

[5] T. K. Vu, M. Bennis, S. Samarakoon, M. Debbah, and M. Latva-aho, "Joint load balancing and interference mitigation in 5G heterogeneous networks," IEEE Trans. Wireless Commun., vol. 16, no. 9, pp. 6032-6046, Sep. 2017.

[6] C. Saha, M. Afshang, and H. S. Dhillon, "Bandwidth partitioning and downlink analysis in millimeter wave integrated access and backhaul for 5G," IEEE Trans. Wireless Commun., vol. 17, no. 12, pp. 8195-8210, Dec. 2018.

[7] M. Polese, M. Giordani, A. Roy, S. Goyal, D. Castor, and M. Zorzi, "Endto-End simulation of integrated access and backhaul at mmWaves," in Proc. IEEE Int. Workshop Comput. Aided Modeling Des. Commun. Links Netw. (CAMAD), Barcelona, Spain, Sep. 2018, pp. 1-7.

[8] M. Mozaffari, W. Saad, M. Bennis, Y. Nam, and M. Debbah, "A tutorial on UAVs for wireless networks: Applications, challenges, and open problems," IEEE Commun. Surveys Tuts., pp. 1-1, Mar. 2019.

[9] M. Mozaffari, W. Saad, M. Bennis, and M. Debbah, "Mobile unmanned aerial vehicles (UAVs) for energy-efficient Internet of Things communications," IEEE Trans. Wireless Commun., vol. 16, no. 11, pp. 7574-7589, Nov. 2017.

[10] J. G. Andrews, S. Buzzi, W. Choi, S. V. Hanly, A. Lozano, A. C. K. Soong, and J. C. Zhang, "What will 5G be?" IEEE J. Sel. Areas Commun. (JSAC), vol. 32, no. 6, pp. 1065-1082, June 2014. 
[11] M. Mozaffari, A. T. Z. Kasgari, W. Saad, M. Bennis, and M. Debbah, "Beyond 5G with UAVs: Foundations of a 3D wireless cellular network," IEEE Trans. Wireless Commun., vol. 18, no. 1, pp. 357-372, Jan. 2019.

[12] M. Chen, M. Mozaffari, W. Saad, C. Yin, M. Debbah, and C. S. Hong, "Caching in the sky: Proactive deployment of cache-enabled unmanned aerial vehicles for optimized quality-of-experience," IEEE J. Sel. Areas Commun. (JSAC), vol. 35, no. 5, pp. 1046-1061, May 2017.

[13] A. Fouda, A. S. Ibrahim, İ. Güvenç, and M. Ghosh, "Interference management in UAV-assisted integrated access and backhaul cellular networks," IEEE Access, pp. 1-1, Jun. 2019.

[14] E. Kalantari, M. Z. Shakir, H. Yanikomeroglu, and A. Yongacoglu, "Backhaulaware robust 3D drone placement in 5G+ wireless networks," in Proc. IEEE Int. Conf. Commun. Workshops (ICC Workshops), Paris, France, May 2017, pp. 109-114.

[15] E. Kalantari, I. Bor-Yaliniz, A. Yongacoglu, and H. Yanikomeroglu, "User association and bandwidth allocation for terrestrial and aerial base stations with backhaul considerations," in Proc. IEEE Int. Symp. Pers., Indoor, Mobile Radio Commun. (PIMRC), Montreal, QC, Canada, Oct. 2017, pp. 1-6.

[16] M. A. Abdel-Malek, A. S. Ibrahim, and M. Mokhtar, "Optimum UAV positioning for better coverage-connectivity tradeoff," in Proc. IEEE Int. Symp. Pers., Indoor, Mobile Radio Commun. (PIMRC), Montreal, QC, Canada, Oct. 2017, pp. 1-5.

[17] A. Fouda, A. S. Ibrahim, İ. Güvenç, and M. Ghosh, "UAV-based in-band integrated access and backhaul for 5G communications," in Proc. IEEE Vehic. Technol.Conf. (VTC-Fall), Chicago, IL, USA, Aug 2018, pp. 1-5.

[18] A. Merwaday, A. Tuncer, A. Kumbhar, and İ. Güvenç, "Improved throughput coverage in natural disasters: Unmanned aerial base stations for public-safety communications," IEEE Veh. Technol. Mag., vol. 11, no. 4, pp. 53-60, Dec. 2016.

[19] D. Athukoralage, İ. Güvenç, W. Saad, and M. Bennis, "Regret based learning for UAV assisted LTE-U/WiFi public safety networks," in Proc. IEEE Global Commun. Conf. (GLOBECOM), Washington, DC, USA, Dec. 2016, pp. 1-7. 
[20] RCR Wireless News, "AT\&T's new flying COW drone to be all-weather disaster insurance," Jun. 2018. [Online]. Available: https://www.rcrwireless.com/20180531/drones/atts-new-flying-cow-droneis-all-weather-disaster-insurance-tag 41.

[21] UAV Coach, "Drone presence at Super Bowl LIII breaks records and sets new precedent for drone use at major public events," Feb. 2019. [Online]. Available: https://uavcoach.com/superbowl-liii-drones/.

[22] J. Garza, M. A. Panduro, A. Reyna, G. Romero, and C. d. Rio, "Design of UAVs-based 3D antenna arrays for a maximum performance in terms of directivity and SLL," Int. J. of Antennas Propag., vol. 2016, no. 2621862, Aug. 2016.

[23] M. Mozaffari, W. Saad, M. Bennis, and M. Debbah, "Communications and control for wireless drone-based antenna array," IEEE Trans. Commun., vol. 67, no. 1, pp. 820-834, Jan. 2019.

[24] U. Challita and W. Saad, "Network formation in the sky: Unmanned aerial vehicles for multi-hop wireless backhauling," in Proc. IEEE Global Commun. Conf. (GLOBECOM), Singapore, Singapore, Dec 2017, pp. 1-6.

[25] J. Stanczak, D. Kozio, I. Z. Kovcs, J. Wigard, M. Wimmer, and R. Amorim, "Enhanced unmanned aerial vehicle communication support in LTE-Advanced," in Proc. IEEE Conf. Standards Commun. Netw. (CSCN), Paris, France, Oct 2018, pp. 1-6.

[26] S. D. Muruganathan, X. Lin, H.-L. Maattanen, Z. Zou, W. A. Hapsari, and S. Yasukawa, "An overview of 3GPP Release-15 study on enhanced LTE support for connected drones," ArXiv e-prints, May 2018. [Online]. Available: https://arxiv.org/abs/1805.00826

[27] N. H. Motlagh, T. Taleb, and O. Arouk, "Low-altitude unmanned aerial vehicles-based Internet of Things services: Comprehensive survey and future perspectives," IEEE Internet Things J., vol. 3, no. 6, pp. 899-922, Dec. 2016.

[28] N. H. Motlagh, M. Bagaa, and T. Taleb, "UAV-based IoT platform: A crowd surveillance use case," IEEE Commun. Mag., vol. 55, no. 2, pp. 128-134, Feb. 2017.

[29] Amazon, "A drone program taking flight," Jun. 2019. [Online]. Available: https://blog.aboutamazon.com/transportation/a-drone-program-taking-flight. 
[30] M. M. Azari, F. Rosas, and S. Pollin, "Cellular connectivity for UAVs: network modeling, performance analysis and design guidelines," IEEE Trans. Wireless Commun., pp. 1-1, Apr. 2019.

[31] G. Geraci, A. Garcia-Rodriguez, L. Galati Giordano, D. Lpez-Prez, and E. Bjrnson, "Understanding UAV cellular communications: From existing networks to massive mimo," IEEE Access, vol. 6, pp. 67853-67865, Nov. 2018.

[32] M. Mozaffari, W. Saad, M. Bennis, and M. Debbah, "Unmanned aerial vehicle with underlaid device-to-device communications: Performance and tradeoffs," IEEE Trans. Wireless Commun., vol. 15, no. 6, pp. 3949-3963, June 2016.

[33] R. I. Bor-Yaliniz, A. El-Keyi, and H. Yanikomeroglu, "Efficient 3-D placement of an aerial base station in next generation cellular networks," in Proc. IEEE Int. Conf. Commun. (ICC), Kuala Lumpur, Malaysia, May 2016, pp. 1-5.

[34] V. Sharma, M. Bennis, and R. Kumar, "UAV-assisted heterogeneous networks for capacity enhancement," IEEE Commun. Lett., vol. 20, no. 6, pp. 1207-1210, June 2016.

[35] W. Guo, C. Devine, and S. Wang, "Performance analysis of micro unmanned airborne communication relays for cellular networks," in Proc. 9th Int. Symp. Commun. Syst., Netw. Digit. Sign (CSNDSP), July 2014, pp. 658-663.

[36] M. Alzenad, A. El-Keyi, F. Lagum, and H. Yanikomeroglu, "3D placement of an unmanned aerial vehicle base station (UAV-BS) for energy-efficient maximal coverage," IEEE Wireless Commun. Lett., vol. 6, no. 4, pp. 434-437, Aug. 2017.

[37] P. G. Sudheesh, M. Mozaffari, M. Magarini, W. Saad, and P. Muthuchidambaranathan, "Sum-Rate analysis for high altitude platform (HAP) drones with tethered balloon relay," IEEE Commun. Lett., vol. 22, no. 6, pp. 12401243, June 2018.

[38] M. Mozaffari, W. Saad, M. Bennis, and M. Debbah, "Optimal transport theory for power-efficient deployment of unmanned aerial vehicles," in Proc. IEEE Int. Conf. Commun. (ICC), May 2016, pp. 1-6.

[39] — - "Wireless communication using unmanned aerial vehicles (UAVs): Optimal transport theory for hover time optimization," IEEE Trans. Wireless Commun., vol. 16, no. 12, pp. 8052-8066, Dec. 2017. 
[40] H. Kim, J. Park, M. Bennis, and S. Kim, "Massive UAV-to-ground communication and its stable movement control: A mean-field approach," in Proc. IEEE 19th Int. Workshop Signal Process. Advances in Wireless Commun. (SPAWC), June 2018, pp. 1-5.

[41] M. Mozaffari, W. Saad, M. Bennis, and M. Debbah, "Efficient deployment of multiple unmanned aerial vehicles for optimal wireless coverage," IEEE Commun. Lett., vol. 20, no. 8, pp. 1647-1650, Aug. 2016.

[42] Q. Zhang, M. Mozaffari, W. Saad, M. Bennis, and M. Debbah, "Machine learning for predictive On-Demand deployment of UAVs for wireless communications," ArXiv e-prints, 2018. [Online]. Available: https: //arxiv.org/abs/1805.00061

[43] L. Wang, Y. L. Che, J. Long, L. Duan, and K. Wu, "Multiple access MmWave design for UAV-aided 5G communications," IEEE Wireless Commun., vol. 26, no. 1, pp. 64-71, Feb. 2019.

[44] Y. Zeng, R. Zhang, and T. J. Lim, "Wireless communications with unmanned aerial vehicles: opportunities and challenges," IEEE Commun. Mag., vol. 54, no. 5, pp. 36-42, May 2016.

[45] T. S. Rappaport, S. Sun, R. Mayzus, H. Zhao, Y. Azar, K. Wang, G. N. Wong, J. K. Schulz, M. Samimi, and F. Gutierrez, "Millimeter wave mobile communications for 5G cellular: It will work!" IEEE Access, vol. 1, pp. 335$349,2013$.

[46] S. Rangan, T. S. Rappaport, and E. Erkip, "Millimeter-Wave cellular wireless networks: Potentials and challenges," Proc. IEEE, vol. 102, no. 3, pp. 366-385, March 2014.

[47] Z. Xiao, P. Xia, and X. Xia, "Enabling UAV cellular with millimeter-wave communication: potentials and approaches," IEEE Commun. Mag., vol. 54, no. 5, pp. 66-73, May 2016.

[48] B. Colo, A. Fouda, and A. S. Ibrahim, "Ray tracing simulations in millimeterwave vehicular communications," in Proc. IEEE Int. Symp. Pers., Indoor, Mobile Radio Commun. (PIMRC), Istanbul, Turkey, Sep. 2019, pp. 1-5.

[49] A. Perez, A. Fouda, and A. S. Ibrahim, "Ray tracing analysis for UAV-assisted integrated access and backhaul millimeter wave networks," in Proc. IEEE 
WoWMoM Workshop Wireless Netw. Planning Comput. UAV Swarms, Washington, DC, USA, Jun. 2019.

[50] N. Rupasinghe, A. S. Ibrahim, and İ. Güvenç, "Optimum hovering locations with angular domain user separation for cooperative UAV networks," in Proc. IEEE Global Commun. Conf. (GLOBECOM), Dec. 2016, pp. 1-6.

[51] N. Rupasinghe, Y. Yapıcı, and İ. Güvenç, "Performance of limited feedback based NOMA transmission in mmWave drone networks," in Proc. IEEE Int. Conf. Commun. Workshops (ICC Workshops), Kansas City, MO, USA, May 2018, pp. 1-6.

[52] N. Rupasinghe, Y. Yapıcı, İ. Güvenç, M. Ghosh, and Y. Kakishima, "Angle feedback for noma transmission in mmwave drone networks," IEEE J. Sel. Topics Signal Process., pp. 1-1, 2019.

[53] Technical Specification Group Radio Access Network, "Study on enhanced LTE support for aerial vehicles," 3GPP, Tech. Rep. 3GPP TR36.777 v15.0.0, Dec. 2017.

[54] E. Kalantari, H. Yanikomeroglu, and A. Yongacoglu, "On the number and 3D placement of drone base stations in wireless cellular networks," in Proc. IEEE Vehic. Technol.Conf. (VTC), Sep. 2016, pp. 1-6.

[55] S. Hanna, H. Yan, and D. Cabric, "Distributed UAV placement optimization for cooperative line-of-sight mimo communications," in Proc. IEEE Int. Conf. Acoust. Speech Signal Process.(ICASSP), Brighton, United Kingdom, May 2019, pp. 4619-4623.

[56] M. Mozaffari, W. Saad, M. Bennis, and M. Debbah, "Drone-based antenna array for service time minimization in wireless networks," in Proc. IEEE Int. Conf. Commun. (ICC), May 2018, pp. 1-6.

[57] L. Sanguinetti, A. L. Moustakas, and M. Debbah, "Interference management in 5G reverse TDD hetnets with wireless backhaul: A large system analysis," IEEE J. Sel. Areas Commun. (JSAC), vol. 33, no. 6, pp. 1187-1200, Jun. 2015.

[58] W. Xia, J. Zhang, S. Jin, C. Wen, F. Gao, and H. Zhu, "Large system analysis of resource allocation in heterogeneous networks with wireless backhaul," IEEE Trans. Commun., vol. 65, no. 11, pp. 5040-5053, Nov. 2017. 
[59] A. D. La Oliva, X. C. Perez, A. Azcorra, A. D. Giglio, F. Cavaliere, D. Tiegelbekkers, J. Lessmann, T. Haustein, A. Mourad, and P. Iovanna, "Xhaul: toward an integrated fronthaul/backhaul architecture in 5G networks," IEEE Wireless Commun., vol. 22, no. 5, pp. 32-40, October 2015.

[60] Y. Liu, A. Tang, and X. Wang, "Joint incentive and resource allocation design for user provided network under $5 \mathrm{G}$ integrated access and backhaul networks," IEEE Trans. Netw. Sci. Eng., pp. 1-1, 2019.

[61] M. N. Islam, N. Abedini, G. Hampel, S. Subramanian, and J. Li, "Investigation of performance in integrated access and backhaul networks," in Proc. IEEE Conf. Comput. Commun. Workshops (INFOCOM WKSHPS), Apr. 2018, pp. 597-602.

[62] W. Pu, X. Li, J. Yuan, and X. Yang, "Resource allocation for millimeter wave self-backhaul network using markov approximation," IEEE Access, vol. 7, pp. 61 283-61 295, 2019.

[63] M. Hashemi, M. Coldrey, M. Johansson, and S. Petersson, "Integrated access and backhaul in fixed wireless access systems," in Proc. IEEE Vehic. Technol.Conf. (VTC-Fall), Sep. 2017, pp. 1-5.

[64] D. Tse and P. Viswanath, Fundamentals of Wireless Communication. Cambridge Univ. Press, 2005.

[65] Technical Specification Group Radio Access Network, "Study on channel model for frequencies from 0.5 to $100 \mathrm{GHz}$," 3GPP, Tech. Rep. 3GPP TR38.901 v15.0.0, Jun. 2018.

[66] M. Ghosh, "A comparison of normalizations for ZF precoded MU-MIMO systems in multipath fading channels," IEEE Wireless Commun. Lett., vol. 2, no. 5, pp. 515-518, Oct. 2013.

[67] T. Yoo and A. Goldsmith, "On the optimality of multiantenna broadcast scheduling using zero-forcing beamforming," IEEE J. Sel. Areas Commun. (JSAC), vol. 24, no. 3, pp. 528-541, Mar. 2006.

[68] J. N. Laneman, D. N. C. Tse, and G. W. Wornell, "Cooperative diversity in wireless networks: Efficient protocols and outage behavior," IEEE Trans. Inf. Theory, vol. 50, no. 12, pp. 3062-3080, Dec. 2004. 
[69] R. Sun, M. Hong, and Z. Luo, "Joint downlink base station association and power control for max-min fairness: Computation and complexity," IEEE J. Sel. Areas Commun. (JSAC), vol. 33, no. 6, pp. 1040-1054, June 2015.

[70] R. Sun and Z. Luo, "Globally optimal joint uplink base station association and power control for max-min fairness," in Proc. IEEE Int. Conf. Acoust. Speech Signal Process.(ICASSP), Florence, Italy, May 2014, pp. 454-458.

[71] J. Kennedy and R. Eberhart, "Particle swarm optimization," in Proc. Int. Conf. Neural Netw. (ICNN), vol. 4, Nov. 1995, pp. 1942-1948 vol.4.

[72] Y. Shi and R. Eberhart, "A modified particle swarm optimizer," in Proc. IEEE Int. Conf. Evol. Comput. IEEE World Congr. on Comput. Intell. (Cat. No.98TH8360), May 1998, pp. 69-73.

[73] D. E. Goldberg, Genetic Algorithms in Search, Optimization and Machine Learning, 1st ed. Boston, MA, USA: Addison-Wesley Longman Publishing Co., Inc., 1989.

[74] Y. Gong, J. Li, Y. Zhou, Y. Li, H. S. Chung, Y. Shi, and J. Zhang, "Genetic learning particle swarm optimization," IEEE Trans. Cybern., vol. 46, no. 10, pp. 2277-2290, Oct. 2016.

[75] J. Plachy, Z. Becvar, P. Mach, R. Marik, and M. Vondra, "Joint positioning of flying base stations and association of users: Evolutionary-based approach," IEEE Access, vol. 7, pp. 11454-11463, Jan. 2019. 